Article

\title{
Coupled Systems of Nonlinear Integer and Fractional Differential Equations with Multi-Point and Multi-Strip Boundary Conditions
}

\author{
Bin $\mathrm{Di}^{1}$, Guo Chen ${ }^{2}$ and Huihui Pang ${ }^{1, *}$ \\ 1 College of Science, China Agricultural University, Beijing 100083, China; caudibin@163.com \\ 2 International College Beijing, China Agricultural University, Beijing 100083, China; \\ GUO.CHEN@ucdenver.edu \\ * Correspondence: phh2000@cau.edu.cn
}

Received: 23 April 2020; Accepted: 1 June 2020; Published: 8 June 2020

\begin{abstract}
We first consider a second order coupled differential system with nonlinearities involved two unknown functions and their derivatives, subject to a new kinds of multi-point and multi-strip boundary value conditions. Since the coupled system contains two dependent variables and their derivatives, the classical method of upper and lower solutions on longer applies. So we adjust and redefine the forms of upper and lower solutions, to establish the existence results. Secondly, we study a Caputo fractional order coupled differential system with discrete multi-point and integral multi-strip boundary value conditions which are very popular recently, and can accurately describe a lot of practical dynamical phenomena, such as control theory, biological system, electroanalytical chemistry and so on. In this part the existence and uniqueness results are achieved via the Leray-Schauder's alternative and the Banach's contraction principle. Finally, an example is presented to illustrate the main results.
\end{abstract}

Keywords: coupled system; multi-point and multi-strip; lower and upper solutions; fractional order; the fixed point theorems

\section{Introduction}

In this paper, we study the existence results to a coupled systems of nonlinear differential equations with multi-point and multi-strip boundary conditions. In the first part, we consider the following second-order coupled differential system

$$
\begin{cases}u^{\prime \prime}(t)+f\left(t, u(t), v(t), u^{\prime}(t), v^{\prime}(t)\right)=0, & t \in(0,1), \\ v^{\prime \prime}(t)+g\left(t, u(t), v(t), u^{\prime}(t), v^{\prime}(t)\right)=0, & t \in(0,1),\end{cases}
$$

equipped with the following boundary value conditions

$$
\left\{\begin{aligned}
u(0)-\sum_{i=1}^{m} \mu_{1 i} u\left(\theta_{1 i}\right) & =\sum_{i=1}^{m} \lambda_{1 i} \int_{0}^{\xi_{1 i}} u(s) d s, \\
u(1)+\sum_{i=1}^{m} \gamma_{1 i} u\left(\vartheta_{1 i}\right) & =\sum_{i=1}^{m} \delta_{1 i} \int_{0}^{\eta_{1 i}} u(s) d s, \\
v(0)-\sum_{i=1}^{m} \mu_{2 i} v\left(\theta_{2 i}\right) & =\sum_{i=1}^{m} \lambda_{2 i} \int_{0}^{\xi_{2 i}} v(s) d s, \\
v(1)+\sum_{i=1}^{m} \gamma_{2 i} v\left(\vartheta_{2 i}\right) & =\sum_{i=1}^{m} \delta_{2 i} \int_{0}^{\eta_{2 i}} v(s) d s,
\end{aligned}\right.
$$


where $0 \leq \theta_{j i}, \xi_{j i}, \vartheta_{j i}, \eta_{j i} \leq 1, \mu_{j i}, \lambda_{j i}$ and $\delta_{j i}$ are nonnegative constants, $\gamma_{j i}$ is negative constant, for $i=1,2, \ldots, m, j=1,2, f, g:[0,1] \times \mathbb{R}^{4} \rightarrow \mathbb{R}$ are continuous. We come to the results by applying the method of upper and lower solutions.

Coupled differential systems are one of the most potential fields since a variety of extensive application in the real word such as physics, chemistry, aerodynamics, rheology, viscoelasticity and so on. For some recent research achievement, readers are referred to a series of books and papers [1-12].

It is generally known that the method of upper and lower solutions is a classical and powerful mechanism that offers existence criterion conditions, for instance, see References [13-22].

In Reference [13], by using the method of upper and lower solutions, authors established an existence results of solutions to a second order coupled differential systems integral boundary value problems in which the nonlinear terms of the system are only related to the unknown functions. Further, in Reference [14], by the same way, authors also studied an existence criterion to a fractional differential equations in which the nonlinear terms depends on unknown functions and its lower derivatives. However, the model in Reference [14] is only a fractional differential equation, not a coupled differential system. Compared with the existing literature [15-17], it is not difficult to find that by using the upper and lower solution method to prove the existence results, the left boundary conditions of boundary value problems discussed are usually equal to zero, which makes straightforward to prove that the solution of the auxiliary truncation function is just between the upper and lower solutions.

In this paper, problem (1) is not only a coupled system with two differential equations in which nonlinear functions depends on all the lower derivative functions, but also boundary conditions (2) is bilateral symmetric, which are nonlocal multi-point and multi-strip boundary conditions. For this reason, problem (1) and (2) is more extensive, meanwhile it leads to more details and difficulties in the proof, which also reflects the value of our conclusion. Accordingly, we will devote our efforts to seek suitable definitions of upper and lower solutions to problem (1) and (2), to establish the criterion of the existence results by the virtue of the Schauder fixed point theorem.

In the second part, we extend our model to the fractional case by considering the following coupled system

$$
\begin{cases}{ }^{c} D_{0+}^{\alpha_{1}} u(t)+f\left(t, u(t), v(t),{ }^{c} D_{0+}^{\alpha_{1}-1} u(t),{ }^{c} D_{0+}^{\alpha_{2}-1} v(t)\right)=0, & t \in(0,1), \\ { }^{c} D_{0+}^{\alpha_{2}} v(t)+g\left(t, u(t), v(t),{ }^{c} D_{0+}^{\alpha_{1}-1} u(t),{ }^{c} D_{0+}^{\alpha_{2}-1} v(t)\right)=0, & t \in(0,1),\end{cases}
$$

with the multi-point fractional derivatives and multi-strip fractional integral boundary conditions

$$
\left\{\begin{array}{l}
u(0)-\sum_{i=1}^{m} \mu_{1 i}{ }^{c} D_{0+}^{q_{1}} u\left(\theta_{1 i}\right)=\sum_{i=1}^{m} \lambda_{1 i} I_{0+}^{p_{1}} u\left(\xi_{1 i}\right), \\
u(1)+\sum_{i=1}^{m} \gamma_{1 i}{ }^{c} D_{0+}^{q_{1}} u\left(\vartheta_{1 i}\right)=\sum_{i=1}^{m} \delta_{1 i} I_{0+}^{p_{1}} u\left(\eta_{1 i}\right), \\
v(0)-\sum_{i=1}^{m} \mu_{2 i}{ }^{c} D_{0+}^{q_{2}} v\left(\theta_{2 i}\right)=\sum_{i=1}^{m} \lambda_{2 i} I_{0+}^{p_{2}} v\left(\xi_{2 i}\right), \\
v(1)+\sum_{i=1}^{m} \gamma_{2 i}{ }^{c} D_{0+}^{q_{2}} v\left(\vartheta_{2 i}\right)=\sum_{i=1}^{m} \delta_{2 i} I_{0+}^{p_{2}} v\left(\eta_{2 i}\right),
\end{array}\right.
$$

where $0 \leq \theta_{j i}, \xi_{j i}, \vartheta_{j i}, \eta_{j i} \leq 1, \mu_{j i}, \lambda_{j i}, \gamma_{j i}$ and $\delta_{j i}$ are nonnegative constants, for $i=1,2, \ldots, m, j=1,2$. ${ }^{c} D_{0+}^{\alpha_{i}},{ }^{c} D_{0+}^{\alpha_{i}-1}$ and ${ }^{c} D_{0+}^{q_{i}}$ are the Caputo fractional derivatives of order $1<\alpha_{i} \leq 2,0<q_{i}<\alpha_{i}-1, p_{i}>0$, $i=1,2 . f, g:[0,1] \times \mathbb{R}^{4} \rightarrow \mathbb{R}$ are continuous.

Fractional order differential systems have been shown to be more realistic and accurate than integer order differential systems [23-33]. Especially, coupled systems of fractional differential equations appear often in investigations connected with anomalous diffusion [27], ecological models [28] and disease models [29-31]. Driven by the wide range of the applications, it is essential to theoretically establish the existence results of solutions. Recently, in Reference [32], Ahmad 
and Ntouyas considered a coupled system of Hadamard type fractional equations. The authors of Reference [33] studied the existence and uniqueness of solutions to a coupled system of nonlinear fractional differential equations with fractional integral conditions.

In Reference [34], authors considered the existence and uniqueness conclusions to the coupled Riemann-Liouville's fractional order differential systems:

$$
\begin{cases}D_{0+}^{\alpha_{1}} u(t)+f_{1}(t, u(t), v(t))=0, & t \in(0,1), \\ D_{0+}^{\alpha_{2}} v(t)+f_{2}(t, u(t), v(t))=0, & t \in(0,1),\end{cases}
$$

with the multi-point and multi-strip integral boundary conditions:

$$
\begin{cases}u(0)=u^{\prime}(0)=0, & u(1)=\sum_{i=1}^{n} \int_{\xi_{i}}^{\eta_{i}} v(s) d A_{i}(s)+\sum_{i=1}^{m-2} b_{i} v\left(\sigma_{i}\right), \\ v(0)=v^{\prime}(0)=0, & v(1)=\sum_{i=1}^{n} \int_{\xi_{i}}^{\eta_{i}} u(s) d A_{i}(s)+\sum_{i=1}^{m-2} b_{i} u\left(\sigma_{i}\right),\end{cases}
$$

where $2<\alpha_{k} \leq 3, D^{\alpha_{k}}$, and $\alpha_{k}, k=1,2 ; 0<\xi_{i}<\eta_{i}<1, A_{i}(s)$ is an nondecreasing function of bounded variation in $[0,1], i=1,2, \ldots, n ; 0<\sigma_{i}<1, b_{i} \geq 0, i=1,2, \ldots, m-2$.

Compared to [34], the couple differential system (3) and (4) is concerned with the Caputo fractional order derivative definition. The equations in (3) are coupled and depend on the two unknown functions and their lower derivatives, the boundary conditions (4) possess the nonlocal form of left and right equilibrium which are different from [34]. Because of the complexity of the form of the problem (3) and (4), we have encountered a lot of resistance in calculating the related Green's functions and discussing their properties. In this part, the existence results are obtained by applying Leray-Schauder's alternative, while the uniqueness of solution is established via Banach's contraction principle.

The structure of this paper is organized as follows. In Section 2, we give some necessary definitions and preliminaries, which are used to prove the existence results to the integer order coupled differential system via the upper and lower solutions method. In Section 3, we establish the existence and uniqueness results of solutions to the fractional order coupled differential system via the Leray-Schauder's alternative, and the Banach's contraction principle. In this section, we illustrate an example to demonstrate the main results. In Section 4, conclusions of this work are outlined.

\section{A Coupled System of Second-Order Differential Equations}

In this section, we consider the existence results of solutions to second-order differential system (1) with multi-point and multi-strip boundary conditions (2). We present here the definitions of upper and lower solutions and Nagumo conditions that will be used to prove our main results.

Definition 1. Functions $(\alpha(t), p(t)),(\beta(t), q(t))$ are called lower solutions and upper solutions of boundary value problem (1.1) and (1.2) if $\alpha(t), p(t), \beta(t)$ and $q(t)$ satisfied:

(1) for $t \in[0,1], \alpha(t) \leq \beta(t), p(t) \leq q(t)$;

(2) for $t \in[0,1], \alpha(t), \beta(t), p(t)$ and $q(t)$ satisfied

$$
\left\{\begin{array}{l}
\alpha^{\prime \prime}(t)+f\left(t, \alpha(t), p(t), \alpha^{\prime}(t),-M_{2}\right) \geq 0 \\
\alpha(0)-\sum_{i=1}^{m} \mu_{1 i} \alpha\left(\theta_{1 i}\right) \leq \sum_{i=1}^{m} \lambda_{1 i} \int_{0}^{\xi_{1 i}} \alpha(s) d s \\
\alpha(1)+\sum_{i=1}^{m} \gamma_{1 i} \alpha\left(\vartheta_{1 i}\right) \leq \sum_{i=1}^{m} \delta_{1 i} \int_{0}^{\eta_{1 i}} \alpha(s) d s
\end{array}\right.
$$




$$
\begin{aligned}
& \left\{\begin{array}{l}
\beta^{\prime \prime}(t)+f\left(t, \beta(t), q(t), \beta^{\prime}(t), M_{2}\right) \leq 0, \\
\beta(0)-\sum_{i=1}^{m} \mu_{1 i} \beta\left(\theta_{1 i}\right) \geq \sum_{i=1}^{m} \lambda_{1 i} \int_{0}^{\xi_{1 i}} \beta(s) d s, \\
\beta(1)+\sum_{i=1}^{m} \gamma_{1 i} \beta\left(\vartheta_{1 i}\right) \geq \sum_{i=1}^{m} \delta_{1 i} \int_{0}^{\eta_{1 i}} \beta(s) d s,
\end{array}\right. \\
& \left\{\begin{array}{l}
p^{\prime \prime}(t)+g\left(t, \alpha(t), p(t),-M_{1}, p^{\prime}(t)\right) \geq 0, \\
p(0)-\sum_{i=1}^{m} \mu_{2 i} p\left(\theta_{2 i}\right) \leq \sum_{i=1}^{m} \lambda_{2 i} \int_{0}^{\xi_{2 i}} p(s) d s, \\
p(1)+\sum_{i=1}^{m} \gamma_{2 i} p\left(\vartheta_{2 i}\right) \leq \sum_{i=1}^{m} \delta_{2 i} \int_{0}^{\eta_{2 i}} p(s) d s,
\end{array}\right. \\
& \left\{\begin{array}{l}
q^{\prime \prime}(t)+g\left(t, \beta(t), q(t), M_{1}, q^{\prime}(t)\right) \leq 0, \\
q(0)-\sum_{i=1}^{m} \mu_{2 i} q\left(\theta_{2 i}\right) \geq \sum_{i=1}^{m} \lambda_{2 i} \int_{0}^{\xi_{2 i}} q(s) d s, \\
q(1)+\sum_{i=1}^{m} \gamma_{2 i} q\left(\vartheta_{2 i}\right) \geq \sum_{i=1}^{m} \delta_{2 i} \int_{0}^{\eta_{2 i}} q(s) d s,
\end{array}\right.
\end{aligned}
$$

where

$$
\begin{aligned}
& M_{1}=\max \left\{\max _{0 \leq t \leq 1}\left|\beta^{\prime}(t)\right|, \max _{0 \leq t \leq 1}\left|\alpha^{\prime}(t)\right|, N_{1}+1\right\}, \\
& M_{2}=\max \left\{\max _{0 \leq t \leq 1}\left|p^{\prime}(t)\right|, \max _{0 \leq t \leq 1}\left|q^{\prime}(t)\right|, N_{2}+1\right\}
\end{aligned}
$$

and

$$
N_{1}=\max _{0 \leq t \leq 1} \beta(t)-\min _{0 \leq t \leq 1} \alpha(t), \quad N_{2}=\max _{0 \leq t \leq 1} q(t)-\min _{0 \leq t \leq 1} p(t) .
$$

Definition 2. Functions $f$ and $g$ are called satisfied Nagumo conditions: if $f\left(t, u(t), v(t), u^{\prime}(t), v^{\prime}(t)\right)$ and $g\left(t, u(t), v(t), u^{\prime}(t), v^{\prime}(t)\right)$ are continuous and the following conditions are satisfied:

$$
\begin{aligned}
& f\left(t, u(t), v(t), u^{\prime}(t), v^{\prime}(t)\right) \leq h_{1}\left(\left|u^{\prime}(t)\right|\right) \\
& g\left(t, u(t), v(t), u^{\prime}(t), v^{\prime}(t)\right) \leq h_{2}\left(\left|v^{\prime}(t)\right|\right)
\end{aligned}
$$

where $h_{i}(s) \in C\left(\mathbb{R}^{+},(0,+\infty)\right), i=1,2$, and

$$
\int_{N_{1}}^{M_{1}} \frac{s}{h_{1}(s)} d s>N_{1}, \int_{N_{2}}^{M_{2}} \frac{s}{h_{2}(s)} d s>N_{2} .
$$

Let us introduce the following hypotheses which are used after:

Hypothesis 1 (H1). $f$ is nondecreasing with respect to the third variable and fifth variable; $g$ is nondecreasing with respect to the second variable and forth variable.

Hypothesis 2 (H2). Problem (1) and (2) has a pair of lower and upper solutions $(\alpha(t), p(t))$ and $(\beta(t), q(t))$, respectively, with $\alpha(t) \leq \beta(t)$ and $p(t) \leq q(t)$, for $t \in[0,1]$.

Hypothesis 3 (H3). $f$ and $g$ satisfy the Nagumo conditions.

Hypothesis 4 (H4). $\mu_{j i}, \lambda_{j i}, \delta_{j i} \geq 0, \gamma_{j i} \leq 0$ and $\sum_{i=1}^{m} \mu_{j i}+\sum_{i=1}^{m} \lambda_{j i} \xi_{j i}<1, \sum_{i=1}^{m} \delta_{j i} \eta_{j i}-\sum_{i=1}^{m} \gamma_{j i}<1$, for $j=1,2$ and $i=1,2, \ldots, m$. 
Considering the following modified differential system:

$$
\begin{cases}u^{\prime \prime}(t)+F\left(t, u(t), v(t), u^{\prime}(t), v^{\prime}(t)\right)=0, & t \in(0,1) \\ v^{\prime \prime}(t)+G\left(t, u(t), v(t), u^{\prime}(t), v^{\prime}(t)\right)=0, & t \in(0,1)\end{cases}
$$

where

$$
\begin{aligned}
& F\left(t, u(t), v(t), u^{\prime}(t), v^{\prime}(t)\right)=f\left(t, r_{1}(u), r_{2}(v), s_{1}\left(u^{\prime}\right), s_{2}\left(v^{\prime}\right)\right)-\frac{u(t)-r_{1}(u)}{1+\left|u(t)-r_{1}(u)\right|} \\
& G\left(t, u(t), v(t), u^{\prime}(t), v^{\prime}(t)\right)=g\left(t, r_{1}(u), r_{2}(v), s_{1}\left(u^{\prime}\right), s_{2}\left(v^{\prime}\right)\right)-\frac{v(t)-r_{2}(v)}{1+\left|v(t)-r_{2}(v)\right|}
\end{aligned}
$$

and

$$
\begin{gathered}
r_{1}(u)=\left\{\begin{array}{rr}
\alpha(t), & u(t)<\alpha(t), \\
u(t), & \alpha(t) \leq u(t) \leq \beta(t), \\
\beta(t), & u(t)>\beta(t),
\end{array} \quad r_{2}(v)=\left\{\begin{array}{lr}
p(t), & v(t)<p(t), \\
v(t), & p(t) \leq v(t) \leq q(t), \\
q(t), & v(t)>q(t),
\end{array}\right.\right. \\
s_{1}\left(u^{\prime}\right)=\left\{\begin{array}{lr}
-M_{1}, & u^{\prime}(t)<-M_{1}, \\
u^{\prime}(t), & \left|u^{\prime}(t)\right| \leq M_{1}, \\
M_{1}, & u^{\prime}(t)>M_{1},
\end{array} \quad s_{2}\left(v^{\prime}\right)=\left\{\begin{array}{lr}
-M_{2}, & v^{\prime}(t)<-M_{2}, \\
v^{\prime}(t), & \left|v^{\prime}(t)\right| \leq M_{2}, \\
M_{2}, & v^{\prime}(t)>M_{2} .
\end{array}\right.\right.
\end{gathered}
$$

Obviously, functions $F$ and $G$ are bounded.

Theorem 1. If conditions H1-H4 hold, then boundary value problem (11) and (2) has at least one pair of solutions $(u(t), v(t))$ such that $\alpha(t) \leq u(t) \leq \beta(t)$ and $p(t) \leq v(t) \leq q(t)$, for $t \in[0,1]$.

Proof. Since $F$ and $G$ defined by (12) are continuous and bounded, applying the Leray-schauder fixed point theorem, we can easily obtain that boundary value problem (11) and (2) has at least one pair of solutions $(u(t), v(t))$. In what follows we need to show that the solutions $(u(t), v(t))$ satisfy $\alpha(t) \leq u(t) \leq \beta(t), p(t) \leq v(t) \leq q(t)$ for $t \in[0,1]$

Supposing that $u(t) \geq \alpha(t)$ on $[0,1]$ is not true, then $\omega(t)=u(t)-\alpha(t)$ has a negative minimum at some $t_{0} \in[0,1]$, that is mean, $\omega\left(t_{0}\right)=\min \{u(t)-\alpha(t) \mid t \in[0,1]\}<0$.

If $t_{0}=0$, then $u(0)<\alpha(0)$. Together (1.2) with the definition of the lower solution, we can get

$$
\begin{aligned}
u(0)-\alpha(0) & \geq \sum_{i=1}^{m} \mu_{1 i} u\left(\theta_{1 i}\right)+\sum_{i=1}^{m} \lambda_{1 i} \int_{0}^{\xi_{1 i}} u(s) d s-\sum_{i=1}^{m} \mu_{1 i} \alpha\left(\theta_{1 i}\right)-\sum_{i=1}^{m} \lambda_{1 i} \int_{0}^{\xi_{1 i}} \alpha(s) d s \\
& =\sum_{i=1}^{m} \mu_{1 i}\left[u\left(\theta_{1 i}\right)-\alpha\left(\theta_{1 i}\right)\right]+\sum_{i=1}^{m} \lambda_{1 i} \int_{0}^{\xi_{1 i}}[u(s)-\alpha(s)] d s \\
& \geq[u(0)-\alpha(0)]\left(\sum_{i=1}^{m} \mu_{1 i}+\sum_{i=1}^{m} \lambda_{1 i} \xi_{1 i}\right)
\end{aligned}
$$

which yields that

$$
[u(0)-\alpha(0)]\left[1-\left(\sum_{i=1}^{m} \mu_{1 i}+\sum_{i=1}^{m} \lambda_{1 i} \xi_{1 i}\right)\right] \geq 0 .
$$

From $\mathrm{H} 4$, we find that there is a contradiction.

If $t_{0} \in(0,1)$, that is, $u\left(t_{0}\right)<\alpha\left(t_{0}\right)$. Obviously, we have $\omega^{\prime \prime}\left(t_{0}\right) \geq 0$ and $\omega^{\prime}\left(t_{0}\right)=0$. Meanwhile, from $\mathrm{H} 1$, we notice 


$$
\begin{aligned}
\omega^{\prime \prime}\left(t_{0}\right)= & u^{\prime \prime}\left(t_{0}\right)-\alpha^{\prime \prime}\left(t_{0}\right) \\
\leq & -F\left(t_{0}, u\left(t_{0}\right), v\left(t_{0}\right), u^{\prime}\left(t_{0}\right), v^{\prime}\left(t_{0}\right)\right)+f\left(t_{0}, \alpha\left(t_{0}\right), p\left(t_{0}\right), \alpha^{\prime}\left(t_{0}\right),-M_{2}\right) \\
= & -f\left(t_{0}, \alpha\left(t_{0}\right), r_{2}\left(v\left(t_{0}\right)\right), \alpha^{\prime}\left(t_{0}\right), s_{2}\left(v^{\prime}\left(t_{0}\right)\right)\right)+\frac{u\left(t_{0}\right)-r_{1}\left(u\left(t_{0}\right)\right)}{1+\left|u\left(t_{0}\right)-r_{1}\left(u\left(t_{0}\right)\right)\right|} \\
& +f\left(t_{0}, \alpha\left(t_{0}\right), p\left(t_{0}\right), \alpha^{\prime}\left(t_{0}\right),-M_{2}\right) \\
< & 0,
\end{aligned}
$$

which contradicts $\omega^{\prime \prime}\left(t_{0}\right) \geq 0$. Thus, we know that the minimum point $t_{0}$ satisfying $u(t)<\alpha(t)$ does not occur on $(0,1)$.

If $t_{0}=1$, then $u(1)<\alpha(1)$. From (2), (5) and H4, we can get

$$
\begin{aligned}
u(1)-\alpha(1) & \geq-\sum_{i=1}^{m} \gamma_{1 i} u\left(\vartheta_{1 i}\right)+\sum_{i=1}^{m} \delta_{1 i} \int_{0}^{\eta_{1 i}} u(s) d s+\sum_{i=1}^{m} \gamma_{1 i} \alpha\left(\vartheta_{1 i}\right)-\sum_{i=1}^{m} \delta_{1 i} \int_{0}^{\eta_{1 i}} \alpha(s) d s \\
& =-\sum_{i=1}^{m} \gamma_{1 i}\left[u\left(\vartheta_{1 i}\right)-\alpha\left(\vartheta_{1 i}\right)\right]+\sum_{i=1}^{m} \delta_{1 i} \int_{0}^{\eta_{1 i}}[u(s)-\alpha(s)] d s \\
& \geq[u(1)-\alpha(1)]\left[\sum_{i=1}^{m} \delta_{1 i} \eta_{1 i}-\sum_{i=1}^{m} \gamma_{1 i}\right],
\end{aligned}
$$

which yields that

$$
[u(1)-\alpha(1)]\left[1-\left(\sum_{i=1}^{m} \delta_{1 i} \eta_{1 i}-\sum_{i=1}^{m} \gamma_{1 i}\right)\right] \geq 0 .
$$

From $\mathrm{H} 4$, we find that there is a contradiction.

Consequently, we finally come to the conclusion that $\alpha(t) \leq u(t)$ for $t \in[0,1]$. Similarly we show $u(t) \leq \beta(t), p(t) \leq v(t) \leq q(t)$ for $t \in[0,1]$.

Theorem 2. If H1-H4 hold, then solutions $(u(t), v(t))$ of boundary value problem (11) and (2) satisfy $\left|u^{\prime}(t)\right| \leq$ $M_{1}$ and $\left|v^{\prime}(t)\right| \leq M_{2}$, where $M_{1}$ and $M_{2}$ defined by (9).

Proof. Now we show that $u^{\prime}(t) \leq M_{1}$ for $t \in[0,1]$.

Suppose that $u^{\prime}(t) \leq M_{1}$ on $[0,1]$ is not true, then there exists $t_{1} \in[0,1]$ such that $u^{\prime}\left(t_{1}\right)>M_{1}$. Due to the fact that $\alpha(t) \leq u(t) \leq \beta(t)$, by the mean value theorem, there exists $t_{2} \in(0,1)$ satisfying

$$
u^{\prime}\left(t_{2}\right)=u(1)-u(0) \leq N_{1}<M_{1}<u^{\prime}\left(t_{1}\right) .
$$

Hence there exist $\left[t_{3}, t_{4}\right] \subset[0,1]$ such that $u^{\prime}\left(t_{3}\right)=N_{1}, u^{\prime}\left(t_{4}\right)=M_{1}$. Further it holds that $N_{1}<u^{\prime}(t)<$ $M_{1}$, for $t \in\left(t_{3}, t_{4}\right)$. From $\mathrm{H} 3$, we have

$$
\left|u^{\prime \prime}(t)\right|=\left|f\left(t, u(t), v(t), u^{\prime}(t), v^{\prime}(t)\right)\right| \leq h_{1}\left(\left|u^{\prime}(t)\right|\right), \text { for } t \in\left(t_{3}, t_{4}\right),
$$

which yields that $\frac{\left|u^{\prime \prime}(t)\right|}{h_{1}\left(\left|u^{\prime}(t)\right|\right)} \leq 1$. Moreover,

$$
\begin{aligned}
& \left|\int_{t_{3}}^{t_{4}} \frac{u^{\prime}(t) u^{\prime \prime}(t)}{h_{1}\left(\left|u^{\prime}(t)\right|\right)} d t\right| \leq \int_{t_{3}}^{t_{4}} \frac{\left|u^{\prime}(t)\right|\left|u^{\prime \prime}(t)\right|}{h_{1}\left(\left|u^{\prime}(t)\right|\right)} d t \\
\leq & \int_{t_{3}}^{t_{4}}\left|u^{\prime}(t)\right| d t=\int_{t_{3}}^{t_{4}} u^{\prime}(t) d t \\
= & u\left(t_{4}\right)-u\left(t_{3}\right) \leq N_{1} .
\end{aligned}
$$

However, according Nagumo condition, it is easy to see that 


$$
\left|\int_{t_{3}}^{t_{4}} \frac{u^{\prime}(t) u^{\prime \prime}(t)}{h_{1}\left(\left|u^{\prime}(t)\right|\right)} d t\right|=\left|\int_{u^{\prime}\left(t_{3}\right)}^{u^{\prime}\left(t_{4}\right)} \frac{u^{\prime}(t)}{h_{1}\left(u^{\prime}(t)\right)} d\left(u^{\prime}(t)\right)\right|=\int_{N_{1}}^{M_{1}} \frac{s}{h_{1}(s)} d s>N_{1} \text {, }
$$

which is a contradiction. Hence, we have that $u^{\prime}(t) \leq M_{1}$ for $t \in[0,1]$.

Similarly, we can show $u^{\prime}(t) \geq-M_{1}$ and $\left|v^{\prime}(t)\right| \leq M_{2}$ for $t \in[0,1]$.

From Theorem 1 and Theorem 2, the solutions of the modified problem (11) and (2) exist and satisfy $\alpha(t) \leq u(t) \leq \beta(t), p(t) \leq v(t) \leq q(t),\left|u^{\prime}(t)\right| \leq M_{1}$ and $\left|v^{\prime}(t)\right| \leq M_{2}$ in the conditions H1-H4. That is to say, the solutions of the modified problem (11) and (2) are the solutions of the original problem (1) and (2).

\section{A Coupled System of Fractional Differential Equations}

In this section, we consider the existence and uniqueness of solution to the fractional differential system (3) with multi-point and multi-strip boundary conditions (4). Some definitions and lemmas are presented here originate from the theory of fractional calculus which will be used for our main theorems.

Definition 3. ([35]) The Riemann-Liouville fractional integral of order $\alpha>0$ of a function $f:(0, \infty) \rightarrow \mathbb{R}$ is given by

$$
I_{0+}^{\alpha} f(t)=\frac{1}{\Gamma(\alpha)} \int_{0}^{t}(t-s)^{\alpha-1} f(s) d s,
$$

provided the right-hand side is pointwise defined on $(0, \infty)$, where $\Gamma(\alpha)$ is the Euler gamma function defined by $\Gamma(\alpha)=\int_{0}^{\infty} t^{\alpha-1} e^{-t} d t$, for $\alpha>0$.

Definition 4. ([29]) The Caputo fractional derivative of order $\alpha>0$ for a function $f:(0, \infty) \rightarrow \mathbb{R}$ is given by

$$
{ }^{c} D_{0+}^{\alpha} f(t)=\frac{1}{\Gamma(n-\alpha)} \int_{0}^{t} f^{(n)}(s)(t-s)^{n-\alpha-1} d s,
$$

where $n=[\alpha]+1$ and $[\alpha]$ stands for the largest integer not greater than $\alpha$.

Lemma 1. Assume that $u \in C[0, \infty)$ with a Caputo fractional derivative of order $\alpha>0$ that belongs to $u \in C^{n}[0, \infty)$, then

$$
I_{0+}^{\alpha}\left({ }^{c} D_{0+}^{\alpha} u(t)\right)=u(t)+c_{1}+c_{2} t+\cdots+c_{n} t^{n-1},
$$

for some $c_{i} \in \mathbb{R}, i=1,2, \ldots, n$.

Lemma 2. Let $\alpha, \beta>0, f \in L^{1}[a, b]$. Then $I_{0+}^{\alpha} I_{0+}^{\beta} f(t)=I_{0+}^{\alpha+\beta} f(t)$ and ${ }^{c} D_{0+}^{\alpha} I_{0+}^{\alpha} f(t)=f(t)$, for all $t \in[a, b]$, where $a, b>0$.

Lemma 3. Let $\beta>\alpha>0, f \in L^{1}[a, b]$. Then ${ }^{c} D_{0+}^{\alpha} I_{0+}^{\beta} f(t)=I_{0+}^{\beta-\alpha} f(t)$, for all $t \in[a, b]$, where $a, b>0$. In addition, this result is always true if the fractional derivative is Riemann-Liouville one.

Lemma 4. For $h(t) \in L^{1}(0,1)$, the solutions of the fractional equation ${ }^{c} D_{0+}^{q} u(t)+h(t)=0,0<t<1$ are

$$
u(t)=-\frac{1}{\Gamma(q)} \int_{0}^{t}(t-s)^{q-1} h(s) d s+c_{1}+c_{2} t+\cdots+c_{n} t^{n-1}, \quad 0<t<1,
$$

where $c_{1}, c_{2}, \ldots, c_{n}$ are arbitrary real constants. 
For convenience, we denote

$$
\begin{aligned}
& D_{j 1}=\frac{1}{\Gamma\left(1+p_{j}\right)} \sum_{i=1}^{m} \lambda_{j i} \xi_{j i}^{p_{j}}, E_{j 1}=\frac{1}{\Gamma\left(2-q_{j}\right)} \sum_{i=1}^{m} \mu_{j i} \theta_{j i}^{1-q_{j}}+\frac{1}{\Gamma\left(2+p_{j}\right)} \sum_{i=1}^{m} \lambda_{j i} \xi_{j i}^{1+p_{j}}, \\
& D_{j 2}=\frac{1}{\Gamma\left(1+p_{j}\right)} \sum_{i=1}^{m} \delta_{j i} \eta_{j i}^{p_{j}}, E_{j 2}=\frac{1}{\Gamma\left(2-q_{j}\right)} \sum_{i=1}^{m} \gamma_{j i} \vartheta_{j i}^{1-q_{j}}-\frac{1}{\Gamma\left(2+p_{j}\right)} \sum_{i=1}^{m} \delta_{j i} \eta_{j i}^{1+p_{j}}, \\
& \Delta_{j}=\left(1+E_{j 2}\right)\left(1-D_{j 1}\right)+E_{j 1}\left(1-D_{j 2}\right),
\end{aligned}
$$

and

$$
\varphi_{j}(t)=\frac{1}{\Delta_{j}}\left[E_{j 1}+\left(1-D_{j 1}\right) t\right], \psi_{j}(t)=\frac{1}{\Delta_{j}}\left[-\left(1+E_{j 2}\right)+\left(1-D_{j 2}\right) t\right], \text { for } t \in[0,1],
$$

where $j=1,2$. In what follows, we always assume that $0<D_{j i}, E_{j i}<1$, for $i, j=1,2$. Obviously, $\Delta_{j}>$ $0, \varphi_{j}(t)>0$, it is easy to get the continuity of $\varphi_{j}(t)$ and $\psi_{j}(t)$, for $j=1,2$.

Lemma 5. Let $1<\alpha_{j}<2,0<q_{j}<\alpha_{j}-1, p_{j}>0$, for $j=1,2$. For $h(t) \in L^{1}(0,1)$, then the following problem

$$
\begin{gathered}
{ }^{c} D_{0+}^{\alpha_{j}} w(t)+h(t)=0, \quad t \in(0,1), \\
w(0)-\sum_{i=1}^{m} \mu_{j i}{ }^{c} D_{0+}^{q_{j}} w\left(\theta_{j i}\right)=\sum_{i=1}^{m} \lambda_{j i} I_{0+}^{p_{j}} w\left(\xi_{j i}\right), \\
w(1)+\sum_{i=1}^{m} \gamma_{j i}{ }^{c} D_{0+}^{q_{j}} w\left(\vartheta_{j i}\right)=\sum_{i=1}^{m} \delta_{j i} I_{0+}^{p_{j}} w\left(\eta_{j i}\right)
\end{gathered}
$$

has an integral representation

$$
w(t)=\int_{0}^{1} G_{j}(t, s) h(s) d s+A_{j}(h) \psi_{j}(t)+B_{j}(h) \varphi_{j}(t)
$$

where

$$
\begin{gathered}
G_{j}(t, s)=\frac{1}{\Gamma\left(\alpha_{j}\right)} \begin{cases}-(t-s)^{\alpha_{j}-1}+\varphi_{j}(t)(1-s)^{\alpha_{j}-1}, & 0 \leq s \leq t \leq 1 \\
\varphi_{j}(t)(1-s)^{\alpha_{j}-1}, & 0 \leq t \leq s \leq 1\end{cases} \\
A_{j}(h)=\sum_{i=1}^{m}\left(\mu_{j i} I_{0+}^{\alpha_{j}-q_{j}} h\left(\theta_{j i}\right)+\lambda_{j i} I_{0+}^{\alpha_{j}+p_{j}} h\left(\xi_{j i}\right)\right) \\
B_{j}(h)=\sum_{i=1}^{m}\left(\gamma_{j i} I_{0+}^{\alpha_{j}-q_{j}} h\left(\vartheta_{j i}\right)-\delta_{j i} I_{0+}^{\alpha_{j}+p_{j}} h\left(\eta_{j i}\right)\right)
\end{gathered}
$$

Proof. According to Lemma 4, (16) can be reduced to the following equivalent integral expression,

$$
w(t)=-I_{0+}^{\alpha_{j}} h(t)+C_{0}+C_{1} t=-\frac{1}{\Gamma\left(\alpha_{j}\right)} \int_{0}^{t}(t-s)^{\alpha_{j}-1} h(s) d s+C_{0}+C_{1} t
$$

where $C_{0}$ and $C_{1}$ are arbitrary real constants.

From (17) with (21), it holds that

$$
\begin{aligned}
& w(0)=C_{0}=\sum_{i=1}^{m} \mu_{j i}{ }^{c} D_{0+}^{q_{j}} w\left(\theta_{j i}\right)+\sum_{i=1}^{m} \lambda_{j i} I_{0+}^{p_{j}} w\left(\xi_{j i}\right), \\
& w(1)=-I_{0+}^{\alpha_{j}} h(1)+C_{0}+C_{1}=-\sum_{i=1}^{m} \gamma_{j i}{ }^{c} D_{0+}^{q_{j}} w\left(\vartheta_{j i}\right)+\sum_{i=1}^{m} \delta_{j i} I_{0+}^{p_{j}} w\left(\eta_{j i}\right) .
\end{aligned}
$$


Since Lemmas 2 and 3, we get

$$
\begin{aligned}
{ }^{c} D_{0+}^{q_{j}} w(t) & =-I_{0+}^{\alpha_{j}-q_{j}} h(t)+C_{1} \frac{t^{1-q_{j}}}{\Gamma\left(2-q_{j}\right)}, \\
I_{0+}^{p_{j}} w(t) & =-I_{0+}^{\alpha_{j}+p_{j}} h(t)+C_{0} \frac{t^{p_{j}}}{\Gamma\left(1+p_{j}\right)}+C_{1} \frac{t^{1+p_{j}}}{\Gamma\left(2+p_{j}\right)} .
\end{aligned}
$$

According to (21)-(23), we can get

$$
\begin{aligned}
& C_{0}=\frac{1}{\Delta_{j}}\left[E_{j 1}\left(I_{0+}^{\alpha_{j}} h(1)+B_{j}(h)\right)-\left(1+E_{j 2}\right) A_{j}(h)\right], \\
& C_{1}=\frac{1}{\Delta_{j}}\left[\left(1-D_{j 1}\right)\left(I_{0+}^{\alpha_{j}} h(1)+B_{j}(h)\right)+\left(1-D_{j 2}\right) A_{j}(h)\right],
\end{aligned}
$$

where $\Delta_{j}$ is introduced by (14), $A_{j}(h), B_{j}(h)$ are denoted by (20).

Taking (24) into (21), we obtain

$$
\begin{aligned}
w(t)= & -I_{0+}^{\alpha_{j}} h(t)+C_{0}+C_{1} t \\
= & -I_{0+}^{\alpha_{j}} h(t)+\frac{1}{\Delta_{j}}\left[E_{j 1}+\left(1-D_{j 1}\right) t\right] I_{0+}^{\alpha_{j}} h(1) \\
& +\frac{1}{\Delta_{j}}\left[E_{j 1}+\left(1-D_{j 1}\right) t\right] B_{j}(h)+\frac{1}{\Delta_{j}}\left[-\left(1+E_{j 2}\right)+\left(1-D_{j 2}\right) t\right] A_{j}(h) \\
= & -I_{0+}^{\alpha_{j}} h(t)+\varphi_{j}(t) I_{0+}^{\alpha_{j}} h(1)+\psi_{j}(t) A_{j}(h)+\varphi_{j}(t) B_{j}(h) \\
= & \int_{0}^{1} G_{j}(t, s) h(s) d s+A_{j}(h) \psi_{j}(t)+B_{j}(h) \varphi_{j}(t),
\end{aligned}
$$

where $G_{j}(t, s), \psi_{j}(t)$ and $\varphi_{j}(t)$ are defined by (19) and (15), respectively.

Lemma 6. For $j=1,2$, the functions $G_{j}(t, s), A_{j}(h)$ and $B_{j}(h)$ admit the following properties:

(1) $\left|G_{j}(t, s)\right| \leq e_{j}(s)$, where $e_{j}(s)=\frac{1}{\Delta_{j} \Gamma\left(\alpha_{j}\right)}(1-s)^{\alpha_{j}-1}\left(\Delta_{j}+E_{j 1}+1-D_{j 1}\right)$, for $t, s \in[0,1]$;

(2) $\left|\frac{\partial}{\partial t} G_{j}(t, s)\right| \leq \bar{e}_{j}$, where $\bar{e}_{j}=\frac{1}{\Delta_{j} \Gamma\left(\alpha_{j}+1\right)}\left(1-D_{j 1}+\alpha_{j} \Delta_{j}\right)$;

(3) $\left|A_{j}(h)\right| \leq \bar{A}_{j}(h),\left|B_{j}(h)\right| \leq \bar{B}_{j}(h)$, for $h(t) \geq 0$, where

$$
\begin{aligned}
& \bar{A}_{j}(h)=\sum_{i=1}^{m} \mu_{j i} I_{0^{+}}^{\alpha_{j}-q_{j}} h(1)+\sum_{i=1}^{m} \lambda_{j i} I_{0^{+}}^{\alpha_{j}+p_{j}} h(1), \\
& \bar{B}_{j}(h)=\sum_{i=1}^{m} \gamma_{j i} I_{0^{+}}^{\alpha_{j}-q_{j}} h(1)+\sum_{i=1}^{m} \delta_{j i} I_{0^{+}}^{\alpha_{j}+p_{j}} h(1) .
\end{aligned}
$$

Proof. (1) For $0 \leq s \leq t \leq 1$, we have

$$
\begin{aligned}
\left|G_{j}(t, s)\right| & =\frac{1}{\Gamma\left(\alpha_{j}\right)}\left|-(t-s)^{\alpha_{j}-1}+\varphi_{j}(t)(1-s)^{\alpha_{j}-1}\right| \\
& \leq \frac{1}{\Gamma\left(\alpha_{j}\right)}\left[(1-s)^{\alpha_{j}-1}+\varphi_{j}(1)(1-s)^{\alpha_{j}-1}\right] \\
& =\frac{1}{\Delta_{j} \Gamma\left(\alpha_{j}\right)}(1-s)^{\alpha_{j}-1}\left[\Delta_{j}+E_{j 1}+1-D_{j 1}\right]=e_{j}(s) .
\end{aligned}
$$


For $0 \leq t \leq s \leq 1$, we have

$$
\begin{aligned}
\left|G_{j}(t, s)\right| & =\frac{1}{\Gamma\left(\alpha_{j}\right)}\left|\varphi_{j}(t)(1-s)^{\alpha_{j}-1}\right| \\
& \leq \frac{1}{\Gamma\left(\alpha_{j}\right)}\left|\left(\varphi_{j}(1)+1\right)(1-s)^{\alpha_{j}-1}\right| \\
& =\frac{1}{\Delta_{j} \Gamma\left(\alpha_{j}\right)}(1-s)^{\alpha_{j}-1}\left[\Delta_{j}+E_{j 1}+1-D_{j 1}\right]=e_{j}(s) .
\end{aligned}
$$

(2) From (19), we have

$$
\begin{aligned}
\left|\frac{\partial}{\partial t} G_{j}(t, s)\right| & \leq \frac{\alpha_{j}-1}{\Gamma\left(\alpha_{j}\right)}(t-s)^{\alpha_{j}-2}+\frac{\left|\varphi_{j}^{\prime}(t)\right|}{\Gamma\left(\alpha_{j}\right)}(1-s)^{\alpha_{j}-1} \\
& =\frac{1}{\Gamma\left(\alpha_{j}+1\right)}\left(\alpha_{j} t^{\alpha_{j}-1}+\left|\varphi_{j}^{\prime}(t)\right|\right) \\
& \leq \frac{1}{\Gamma\left(\alpha_{j}+1\right)}\left(\frac{1-D_{j 1}}{\Delta_{j}}+\alpha_{j}\right)=\bar{e}_{j} .
\end{aligned}
$$

(3) From (20), for $h(t) \geq 0$, we have

$$
\begin{aligned}
& \left|A_{j}(h)\right| \leq \sum_{i=1}^{m}\left|\mu_{j i} I_{0+}^{\alpha_{j}-q_{j}} h\left(\theta_{j i}\right)+\lambda_{j i} I_{0+}^{\alpha_{j}+p_{j}} h\left(\xi_{j i}\right)\right| \\
= & \sum_{i=1}^{m}\left(\frac{\mu_{j i}}{\Gamma\left(\alpha_{j}-q_{j}\right)} \int_{0}^{\theta_{j i}}\left(\theta_{j i}-s\right)^{\alpha_{j}-q_{j}-1} h(s) d s+\frac{\lambda_{j i}}{\Gamma\left(\alpha_{j}+p_{j}\right)} \int_{0}^{\xi_{j i}}\left(\xi_{j i}-s\right)^{\alpha_{j}+p_{j}-1} h(s) d s\right) \\
\leq & \sum_{i=1}^{m}\left(\frac{\mu_{j i}}{\Gamma\left(\alpha_{j}-q_{j}\right)} \int_{0}^{1}(1-s)^{\alpha_{j}-q_{j}-1} h(s) d s+\frac{\lambda_{j i}}{\Gamma\left(\alpha_{j}+p_{j}\right)} \int_{0}^{1}(1-s)^{\alpha_{j}+p_{j}-1} h(s) d s\right) \\
= & \bar{A}_{j}(h) .
\end{aligned}
$$

Similarly, for $h(t) \geq 0$, we have

$$
\begin{aligned}
& \left|B_{j}(h)\right| \leq \sum_{i=1}^{m}\left|\gamma_{j i} I_{0+}^{\alpha_{j}-q_{j}} h\left(\vartheta_{j i}\right)-\delta_{j i} I_{0+}^{\alpha_{j}+p_{j}-1} h\left(\eta_{j i}\right)\right| \\
= & \sum_{i=1}^{m}\left|\frac{\gamma_{j i}}{\Gamma\left(\alpha_{j}-q_{j}\right)} \int_{0}^{\vartheta_{j i}}\left(\vartheta_{j i}-s\right)^{\alpha_{j}-q_{j}-1} h(s) d s-\frac{\delta_{j i}}{\Gamma\left(\alpha_{j}+p_{j}\right)} \int_{0}^{\eta_{j i}}\left(\eta_{j i}-s\right)^{\alpha_{j}+p_{j}-1} h(s) d s\right| \\
\leq & \sum_{i=1}^{m}\left(\frac{\gamma_{j i}}{\Gamma\left(\alpha_{j}-q_{j}\right)} \int_{0}^{1}(1-s)^{\alpha_{j}-q_{j}-1} h(s) d s+\frac{\delta_{j i}}{\Gamma\left(\alpha_{j}+p_{j}\right)} \int_{0}^{1}(1-s)^{\alpha_{j}+p_{j}-1} h(s) d s\right) \\
= & \bar{B}_{j}(h) .
\end{aligned}
$$

Lemma 7. (the Leray-Schauder's Alternative) Let $F: E \rightarrow E$ be a completely continuous operator (i.e., a map that restricted to any bounded set in $E$ is compact). Let

$$
\varepsilon(F)=\{x \in E: x=\lambda F(x), 0<\lambda<1\} .
$$

Then either the set $\varepsilon(F)$ is unbounded, or $F$ has at least one fixed point.

Define the space $X=\left\{u(t) \mid u(t) \in C[0,1]\right.$ and $\left.{ }^{c} D_{0+}^{\alpha_{1}-1} u(t) \in C[0,1]\right\}$ endowed with the norm $\|u\|_{X}=\max _{t \in[0,1]}|u(t)|+\max _{t \in[0,1]}\left|{ }^{c} D_{0+}^{\alpha_{1}-1} u(t)\right|$, and $Y=\left\{v(t) \mid v(t) \in C[0,1]\right.$ and $\left.{ }^{c} D_{0+}^{\alpha_{2}-1} v(t) \in C[0,1]\right\}$ 
endowed with the norm $\|v\|_{Y}=\max _{t \in[0,1]}|v(t)|+\max _{t \in[0,1]}\left|{ }^{c} D_{0+}^{\alpha_{2}-1} v(t)\right|$. It is easy to see that $\left(X,\|\cdot\|_{X}\right)$ and $\left(Y,\|\cdot\|_{Y}\right)$ are Banach spaces. Further, the product space $\left(X \times Y,\|(u, v)\|_{X \times Y}\right)$ is also a Banach space with the norm $\|(u, v)\|_{X \times Y}=\|u\|_{X}+\|v\|_{Y}$.

Let $T: X \times Y \rightarrow X \times Y$ be the operator defined by

$$
T(u, v)(t)=\left(T_{1}(u, v)(t), T_{2}(u, v)(t)\right)
$$

where

$$
\begin{aligned}
T_{1}(u, v)(t)= & \int_{0}^{1} G_{1}(t, s) f\left(s, u(s), v(s),{ }^{c} D_{0+}^{\alpha_{1}-1} u(s),{ }^{c} D_{0+}^{\alpha_{2}-1} v(s)\right) d s \\
& +A_{1}\left(f_{s}(u, v)\right) \psi_{1}(t)+B_{1}\left(f_{s}(u, v)\right) \varphi_{1}(t), \\
T_{2}(u, v)(t)= & \int_{0}^{1} G_{2}(t, s) g\left(s, u(s), v(s),{ }^{c} D_{0+}^{\alpha_{1}-1} u(s),{ }^{c} D_{0+}^{\alpha_{2}-1} v(s)\right) d s \\
& +A_{2}\left(g_{s}(u, v)\right) \psi_{2}(t)+B_{2}\left(g_{s}(u, v)\right) \varphi_{2}(t) .
\end{aligned}
$$

Lemma 8. $T: X \times Y \rightarrow X \times Y$ is completely continuous.

Proof. The operator $T$ is continuous owing to the continuities of $G_{j}(t, s), A_{1}(f), B_{1}(f), A_{2}(g), B_{2}(g)$, $\varphi_{j}(t), \psi_{j}(t)$, for $j=1,2, f$ and $g$.

Denote $\Omega \subset X \times Y$ is a bounded set. Hence there is a positive constant $L$ such that

$$
\left|f\left(t, u(t), v(t),{ }^{c} D_{0+}^{\alpha_{1}-1} u(t),{ }^{c} D_{0+}^{\alpha_{2}-1} v(t)\right)\right| \leq L, \text { for }(u, v) \in \Omega .
$$

For convenience, we denote $\psi_{1 M}=\max \left\{\left|\psi_{1}(0)\right|,\left|\psi_{1}(1)\right|\right\}$. For any $(u, v) \in \Omega$, we get

$$
\begin{aligned}
\left|T_{1}(u, v)(t)\right| & =\left|\int_{0}^{1} G_{1}(t, s) f\left(s, u(s), v(s),{ }^{c} D_{0+}^{\alpha_{1}-1} u(s),{ }^{c} D_{0+}^{\alpha_{2}-1} v(s)\right) d s+A_{1}(f) \psi_{1}(t)+B_{1}(f) \varphi_{1}(t)\right| \\
& \leq\left|\int_{0}^{1} e_{1}(s) f\left(s, u(s), v(s),{ }^{c} D_{0+}^{\alpha_{1}-1} u(s),{ }^{c} D_{0+}^{\alpha_{2}-1} v(s)\right) d s\right|+\left|A_{1}(f) \psi_{1}(t)\right|+\left|B_{1}(f) \varphi_{1}(t)\right| \\
& \leq L \frac{\varphi_{1}(1)+1}{\Gamma\left(\alpha_{1}+1\right)}+L \bar{A}_{1}(1) \psi_{1 M}+L \bar{B}_{1}(1) \varphi_{1}(1), \\
\left|T_{1}^{\prime}(u, v)(t)\right| & =\left|\int_{0}^{1} \frac{\partial}{\partial t} G_{1}(t, s) f\left(s, u(s), v(s),{ }^{c} D_{0+}^{\alpha_{1}-1} u(s),{ }^{c} D_{0+}^{\alpha_{2}-1} v(s)\right) d s+A_{1}(f) \psi_{1}^{\prime}(t)+B_{1}(f) \varphi_{1}^{\prime}(t)\right| \\
& \leq\left|\int_{0}^{1} \bar{e}_{1} f\left(s, u(s), v(s),{ }^{c} D_{0+}^{\alpha_{1}-1} u(s),{ }^{c} D_{0+}^{\alpha_{2}-1} v(s)\right) d s\right|+\left|A_{1}(f) \psi_{1}^{\prime}(t)\right|+\left|B_{1}(f) \varphi_{1}^{\prime}(t)\right| \\
& \leq L \bar{e}_{1}+L \bar{A}_{1}(1) \frac{1-D_{12}}{\Delta_{1}}+L \bar{B}_{1}(1) \frac{1-D_{11}}{\Delta_{1}} .
\end{aligned}
$$

Further, we have

$$
\begin{aligned}
\left|{ }^{c} D_{0+}^{\alpha_{1}-1} T_{1}(u, v)(t)\right| & \leq \int_{0}^{t} \frac{(t-s)^{1-\alpha_{1}}}{\Gamma\left(2-\alpha_{1}\right)}\left|T_{1}^{\prime}(u, v)(s)\right| d s \\
& \leq \frac{t^{2-\alpha_{1}}}{\Gamma(3-\alpha)}\left(L \bar{e}_{1}+L \bar{A}_{1}(1) \frac{1-D_{12}}{\Delta_{1}}+L \bar{B}_{1}(1) \frac{1-D_{11}}{\Delta_{1}}\right) \\
& \leq \frac{1}{\Gamma\left(3-\alpha_{1}\right)}\left(L \bar{e}_{1}+L \bar{A}_{1}(1) \frac{1-D_{12}}{\Delta_{1}}+L \bar{B}_{1}(1) \frac{1-D_{11}}{\Delta_{1}}\right)
\end{aligned}
$$


which implies that

$$
\begin{aligned}
\left\|T_{1}(u, v)(t)\right\|_{X}= & \max _{t \in[0,1]}\left|T_{1}(u, v)(t)\right|+\max _{t \in[0,1]}\left|{ }^{c} D_{0+}^{\alpha_{1}-1} T_{1}(u, v)(t)\right| \\
\leq & L \frac{\varphi_{1}(1)+1}{\Gamma\left(\alpha_{1}+1\right)}+L \bar{A}_{1}(1) \psi_{1^{M}}+L \bar{B}_{1}(1) \varphi_{1}(1) \\
& +\frac{1}{\Gamma\left(3-\alpha_{1}\right)}\left(L \bar{e}_{1}+L \bar{A}_{1}(1) \frac{1-D_{12}}{\Delta_{1}}+L \bar{B}_{1}(1) \frac{1-D_{11}}{\Delta_{1}}\right) .
\end{aligned}
$$

Therefore, the above inequalities implies that the operator $T_{1}$ is uniformly bounded. In a similar manner, $T_{2}$ is also uniformly bounded. Thus, the operator $T$ is uniformly bounded.

Next step, we prove that $T$ is equicontinuous. Let $t_{1}, t_{2} \in[0,1]$ with $t_{1} \leq t_{2}$. Then we get

$$
\begin{aligned}
\left|T_{1}(u, v)\left(t_{2}\right)-T_{1}(u, v)\left(t_{1}\right)\right| & =\left|\int_{t_{1}}^{t_{2}} T_{1}^{\prime}(u, v)(s) d s\right| \leq \int_{t_{1}}^{t_{2}}\left|T_{1}^{\prime}(u, v)(s)\right| d s \\
& \leq\left(L \bar{e}_{1}+L \bar{A}_{1}(1) \frac{1-D_{12}}{\Delta_{1}}+L \bar{B}_{1}(1) \frac{1-D_{11}}{\Delta_{1}}\right)\left(t_{2}-t_{1}\right)
\end{aligned}
$$

and

$$
\begin{aligned}
& \left|{ }^{c} D_{0+}^{\alpha_{1}-1} T_{1}(u, v)\left(t_{2}\right)-{ }^{c} D_{0+}^{\alpha_{1}-1} T_{1}(u, v)\left(t_{1}\right)\right| \\
= & \frac{1}{\Gamma\left(2-\alpha_{1}\right)}\left|\int_{0}^{t_{2}}\left(t_{2}-s\right)^{1-\alpha_{1}} T_{1}^{\prime}(u, v)(s) d s-\int_{0}^{t_{1}}\left(t_{1}-s\right)^{1-\alpha_{1}} T_{1}^{\prime}(u, v)(s) d s\right| \\
\leq & \frac{1}{\Gamma\left(2-\alpha_{1}\right)}\left|\int_{0}^{t_{2}}\left(t_{2}-s\right)^{1-\alpha_{1}} T_{1}^{\prime}(u, v)(s) d s-\int_{0}^{t_{1}}\left(t_{2}-s\right)^{1-\alpha_{1}} T_{1}^{\prime}(u, v)(s) d s\right| \\
& +\frac{1}{\Gamma\left(2-\alpha_{1}\right)}\left|\int_{0}^{t_{1}}\left(t_{2}-s\right)^{1-\alpha_{1}} T_{1}^{\prime}(u, v)(s) d s-\int_{0}^{t_{1}}\left(t_{1}-s\right)^{1-\alpha_{1}} T_{1}^{\prime}(u, v)(s) d s\right| \\
\leq & \frac{1}{\Gamma\left(2-\alpha_{1}\right)}\left|\int_{t_{1}}^{t_{2}}\left(t_{2}-s\right)^{1-\alpha_{1}}\right| T_{1}^{\prime}(u, v)(s)|d s| \\
& +\frac{1}{\Gamma\left(2-\alpha_{1}\right)}\left|\int_{0}^{t_{1}}\left(\left(t_{2}-s\right)^{1-\alpha_{1}}-\left(t_{1}-s\right)^{1-\alpha_{1}}\right)\right| T_{1}^{\prime}(u, v)(s)|d s| \\
\leq & \frac{L}{\Gamma\left(2-\alpha_{1}\right)}\left(\bar{e}_{1}+\bar{A}_{1}(1) \frac{1-D_{12}}{\Delta_{1}}+\bar{B}_{1}(1) \frac{1-D_{11}}{\Delta_{1}}\right) \\
& \times\left(\left|\int_{t_{1}}^{t_{2}}\left(t_{2}-s\right)^{1-\alpha_{1}} d s\right|+\left|\int_{0}^{t_{1}}\left(\left(t_{2}-s\right)^{1-\alpha_{1}}-\left(t_{1}-s\right)^{1-\alpha_{1}}\right) d s\right|\right) \\
\leq & \frac{L}{\Gamma\left(3-\alpha_{1}\right)}\left(\bar{e}_{1}+\bar{A}_{1}(1) \frac{1-D_{12}}{\Delta_{1}}+\bar{B}_{1}(1) \frac{1-D_{11}}{\Delta_{1}}\right)\left(2\left(t_{2}-t_{1}\right)^{2-\alpha_{1}}+t_{2}^{2-\alpha_{1}}-t_{1}^{2-\alpha_{1}}\right) .
\end{aligned}
$$

So, we can get

$$
\begin{aligned}
& \left\|T_{1}(u, v)\left(t_{2}\right)-T_{1}(u, v)\left(t_{1}\right)\right\|_{X} \\
\leq & \left(L \bar{e}_{1}+L \bar{A}_{1}(1) \frac{1-D_{12}}{\Delta_{1}}+L \bar{B}_{1}(1) \frac{1-D_{11}}{\Delta_{1}}\right)\left(t_{2}-t_{1}\right) \\
& +\frac{L}{\Gamma\left(3-\alpha_{1}\right)}\left(\bar{e}_{1}+\bar{A}_{1}(1) \frac{1-D_{12}}{\Delta_{1}}+\bar{B}_{1}(1) \frac{1-D_{11}}{\Delta_{1}}\right)\left(2\left(t_{2}-t_{1}\right)^{2-\alpha_{1}}+t_{2}^{2-\alpha_{1}}-t_{1}^{2-\alpha_{1}}\right),
\end{aligned}
$$

which implied that $\left\|T_{1}(u, v)\left(t_{2}\right)-T_{1}(u, v)\left(t_{1}\right)\right\|_{X} \rightarrow 0$ as $t_{2} \rightarrow t_{1}$. In a similar way, we can obtain that $\left\|T_{2}(u, v)\left(t_{2}\right)-T_{2}(u, v)\left(t_{1}\right)\right\|_{Y} \rightarrow 0$ as $t_{2} \rightarrow t_{1}$. Therefore, the operator $T$ is equicontinuous. Thus $T$ is completely continuous. 
Let us give some assumptions which are used later.

Hypothesis 5 (H5). Suppose that there is real constants $\rho_{0} \geq 0, \varrho_{0} \geq 0, \rho_{i}>0$ and $\varrho_{i}>0, i=1,2,3,4$, such that for $t \in[0,1]$ and $x_{i} y_{i} \in \mathbb{R}$, we have

$$
\begin{aligned}
& \left|f\left(t, x_{1}, x_{2}, x_{3}, x_{4}\right)\right| \leq \rho_{0}+\rho_{1}\left|x_{1}\right|+\rho_{2}\left|x_{2}\right|+\rho_{3}\left|x_{3}\right|+\rho_{4}\left|x_{4}\right| \\
& \left|g\left(t, y_{1}, y_{2}, y_{3}, y_{4}\right)\right| \leq \varrho_{0}+\varrho_{1}\left|y_{1}\right|+\varrho_{2}\left|y_{2}\right|+\varrho_{3}\left|y_{3}\right|+\varrho_{4}\left|y_{4}\right| .
\end{aligned}
$$

Hypothesis 6 (H6). Suppose that $f, g:[0,1] \times \mathbb{R}^{4} \rightarrow \mathbb{R}$ are continuous, and there are real constants $n_{i}, m_{i}, i=1,2,3,4$, such that for all $t \in[0,1]$ and $u_{1}, v_{1} \in \mathbb{R}$, we have

$$
\begin{aligned}
& \left|f\left(t, u_{1}, u_{2}, u_{3}, u_{4}\right)-f\left(t, v_{1}, v_{2}, v_{3}, v_{4}\right)\right| \\
\leq & n_{1}\left|u_{1}-v_{1}\right|+n_{2}\left|u_{2}-v_{2}\right|+n_{3}\left|u_{3}-v_{3}\right|+n_{4}\left|u_{4}-v_{4}\right|, \\
& \left|g\left(t, u_{1}, u_{2}, u_{3}, u_{4}\right)-g\left(t, v_{1}, v_{2}, v_{3}, v_{4}\right)\right| \\
\leq & m_{1}\left|u_{1}-v_{1}\right|+m_{2}\left|u_{2}-v_{2}\right|+m_{3}\left|u_{3}-v_{3}\right|+m_{4}\left|u_{4}-v_{4}\right| .
\end{aligned}
$$

Set

$$
\begin{aligned}
& \bar{N}_{1}= \frac{\varphi_{1}(1)+1}{\Gamma\left(\alpha_{1}+1\right)}+\bar{A}_{1}(1) \psi_{1} M+\bar{B}_{1}(1) \varphi_{1}(1) \\
&+\frac{1}{\Gamma\left(3-\alpha_{1}\right)}\left(\bar{e}_{1}+\bar{A}_{1}(1) \frac{1-D_{12}}{\Delta_{1}}+\bar{B}_{1}(1) \frac{1-D_{11}}{\Delta_{1}}\right), \\
& \bar{N}_{2}= \frac{\varphi_{2}(1)+1}{\Gamma\left(\alpha_{2}+1\right)}+\bar{A}_{2}(1) \psi_{2^{M}}+\bar{B}_{2}(1) \varphi_{2}(1) \\
&+\frac{1}{\Gamma\left(3-\alpha_{2}\right)}\left(\bar{e}_{2}+\bar{A}_{2}(1) \frac{1-D_{22}}{\Delta_{2}}+\bar{B}_{2}(1) \frac{1-D_{21}}{\Delta_{2}}\right), \\
& N_{3}=\max \left\{\bar{N}_{1} \max \left\{\rho_{1}, \rho_{3}\right\}+\bar{N}_{2} \max \left\{\varrho_{1}, \varrho_{3}\right\}, \bar{N}_{1} \max \left\{\rho_{2}, \rho_{4}\right\}+\bar{N}_{2} \max \left\{\varrho_{2}, \varrho_{4}\right\}\right\} . \\
& M_{3}=\max \left\{\bar{N}_{1} \max \left\{n_{1}, n_{3}\right\}+\bar{N}_{2} \max \left\{m_{1}, m_{3}\right\}, \bar{N}_{1} \max \left\{n_{2}, n_{4}\right\}+\bar{N}_{2} \max \left\{m_{2}, m_{4}\right\}\right\} .
\end{aligned}
$$

The following conclusion is based on the Leray-Schauder's alternative.

Theorem 3. Suppose that H5 is satisfied. In addition, suppose that $N_{3}<1$. Then the boundary value problem (3) and (4) has at least one solution.

Proof. It will be checked that $\varepsilon=\{(u, v) \in X \times Y \mid(u, v)=\lambda T(u, v), 0 \leq \lambda \leq 1\}$ is bounded. Let $(u, v) \in \varepsilon$, then $(u, v)=\lambda T(u, v)$. For $t \in[0,1]$, we obtain

$$
u(t)=\lambda T_{1}(u, v)(t) .
$$

From H5, we have 


$$
\begin{aligned}
|u(t)|= & \lambda\left|T_{1}(u, v)(t)\right| \\
\leq & \left|\int_{0}^{1} G_{1}(t, s) f\left(s, u(s), v(s),{ }^{c} D_{0+}^{\alpha_{1}-1} u(s),{ }^{c} D_{0+}^{\alpha_{2}-1} v(s)\right) d s+A_{1}(f) \psi_{1}(t)+B_{1}(f) \varphi_{1}(t)\right| \\
\leq & \int_{0}^{1}\left|G_{1}(t, s) f\left(s, u(s), v(s),{ }^{c} D_{0+}^{\alpha_{1}-1} u(s),{ }^{c} D_{0+}^{\alpha_{2}-1} v(s)\right)\right| d s+\left|A_{1}(f) \psi_{1}(t)\right|+\left|B_{1}(f) \varphi_{1}(t)\right| \\
\leq & \left(\rho_{0}+\rho_{1}|u(t)|+\rho_{2}|v(t)|+\rho_{3}\left|{ }^{c} D_{0+}^{\alpha_{1}-1} u(t)\right|+\left.\rho_{4}\right|^{c} D_{0+}^{\alpha_{2}-1} v(t) \mid\right) \\
& \times\left[\int_{0}^{1} e_{1}(s) d s+\bar{A}_{1}(1) \psi_{1}{ }^{M}+\bar{B}_{1}(1) \varphi_{1}(1)\right] \\
\leq & \left(\rho_{0}+\max \left\{\rho_{1}, \rho_{3}\right\}\|u\|_{X}+\max \left\{\rho_{2}, \rho_{4}\right\}\|v\|_{Y}\right)\left[\frac{\varphi_{1}(1)+1}{\Gamma\left(\alpha_{1}+1\right)}+\bar{A}_{1}(1) \psi_{1}{ }^{M}+\bar{B}_{1}(1) \varphi_{1}(1)\right], \\
\left|u^{\prime}(t)\right|= & \lambda\left|T_{1}^{\prime}(u, v)(t)\right| \\
\leq & \left|\int_{0}^{1} \frac{\partial}{\partial t} G_{1}(t, s) f\left(s, u(s), v(s),{ }^{c} D_{0+}^{\alpha_{1}-1} u(s),{ }^{c} D_{0+}^{\alpha_{2}-1} v(s)\right) d s+A_{1}(f) \psi_{1}^{\prime}(t)+B_{1}(f) \varphi_{1}^{\prime}(t)\right| \\
\leq & \left(\rho_{0}+\max \left\{\rho_{1}, \rho_{3}\right\}\|u\|_{X}+\max \left\{\rho_{2}, \rho_{4}\right\}\|v\|_{Y}\right)\left[\bar{e}_{1}+\bar{A}_{1}(1) \frac{1-D_{12}}{\Delta_{1}}+\bar{B}_{1}(1) \frac{1-D_{11}}{\Delta_{1}}\right],
\end{aligned}
$$

and

$$
\begin{aligned}
\left|{ }^{c} D_{0+}^{\alpha_{1}-1} u(t)\right|= & \lambda\left|{ }^{c} D_{0+}^{\alpha_{1}-1} T_{1}(u, v)(t)\right| \\
\leq & \left|\int_{0}^{t} \frac{(t-s)^{1-\alpha_{1}}}{\Gamma\left(2-\alpha_{1}\right)} T_{1}^{\prime}(u, v)(s) d s\right| \\
\leq & \left(\rho_{0}+\max \left\{\rho_{1}, \rho_{3}\right\}\|u\|_{X}+\max \left\{\rho_{2}, \rho_{4}\right\}\|v\|_{Y}\right) \\
& \times \frac{1}{\Gamma\left(3-\alpha_{1}\right)}\left[\bar{e}_{1}+\bar{A}_{1}(1) \frac{1-D_{12}}{\Delta_{1}}+\bar{B}_{1}(1) \frac{1-D_{11}}{\Delta_{1}}\right] .
\end{aligned}
$$

Hence we have

$$
\begin{aligned}
\|u\|_{X} \leq & \left(\rho_{0}+\max \left\{\rho_{1}, \rho_{3}\right\}\|u\|_{X}+\max \left\{\rho_{2}, \rho_{4}\right\}\|v\|_{Y}\right)\left[\frac{\varphi_{1}(1)+1}{\Gamma\left(\alpha_{1}+1\right)}+\bar{A}_{1}(1) \psi_{1 M}\right. \\
& \left.+\bar{B}(1) \varphi_{1}(1)+\frac{1}{\Gamma\left(3-\alpha_{1}\right)}\left(\bar{e}_{1}+\bar{A}_{1}(1) \frac{1-D_{12}}{\Delta_{1}}+\bar{B}_{1}(1) \frac{1-D_{11}}{\Delta_{1}}\right)\right] \\
= & \left(\rho_{0}+\max \left\{\rho_{1}, \rho_{3}\right\}\|u\|_{X}+\max \left\{\rho_{2}, \rho_{4}\right\}\|v\|_{Y}\right) \bar{N}_{1} .
\end{aligned}
$$

Similarly, we can get

$$
\|v\|_{Y} \leq\left(\varrho_{0}+\max \left\{\varrho_{1}, \varrho_{3}\right\}\|u\|_{X}+\max \left\{\varrho_{2}, \varrho_{4}\right\}\|v\|_{Y}\right) \bar{N}_{2} .
$$

Combining (30) with (31), we obtain

$$
\begin{aligned}
\|(u, v)\|_{X \times Y}= & \|u\|_{X}+\|v\|_{Y} \\
\leq & \left(\rho_{0}+\max \left\{\rho_{1}, \rho_{3}\right\}\|u\|_{X}+\max \left\{\rho_{2}, \rho_{4}\right\}\|v\|_{Y}\right) \bar{N}_{1} \\
& +\left(\varrho_{0}+\max \left\{\varrho_{1}, \varrho_{3}\right\}\|u\|_{X}+\max \left\{\varrho_{2}, \varrho_{4}\right\}\|v\|_{Y}\right) \bar{N}_{2} \\
\leq & N_{3}\left(\|u\|_{X}+\|v\|_{Y}\right)+\rho_{0} \bar{N}_{1}+\varrho_{0} \bar{N}_{2} \\
= & N_{3}\|(u, v)\|_{X \times Y}+\rho_{0} \bar{N}_{1}+\varrho_{0} \bar{N}_{2}
\end{aligned}
$$


as a result

$$
\|(u, v)\|_{X \times Y} \leq \frac{\rho_{0} \bar{N}_{1}+\varrho_{0} \bar{N}_{2}}{1-N 3}
$$

where $\bar{N}_{1}, \bar{N}_{2}$ and $N_{3}$ are defined by (27) and (28), which show that the set $\varepsilon$ is bounded. Therefore, from Lemma 7, $T$ has at least one fixed point which implies problem (3) and (4) has at least one pair of solutions.

In what follows, we show the uniqueness result of solutions to problem (3) and (4) based on the Banach's contraction principle.

Theorem 4. Suppose that H6 holds. In addition, suppose that $M_{3}<1$. Then problem (3) and (4) has a unique pair of solutions.

Proof. Define

$$
\begin{aligned}
& \sup _{t \in[0,1]}|f(t, 0,0,0,0)|=H_{1}<\infty, \\
& \sup _{t \in[0,1]}|g(t, 0,0,0,0)|=H_{2}<\infty,
\end{aligned}
$$

such that

$$
r \geq \frac{\bar{N}_{1} H_{1}+\bar{N}_{2} H_{2}}{1-M_{3}} .
$$

We show that $T B_{r} \subset B_{r}$, where $B_{r}=\left\{(u, v) \in X \times Y:\|(u, v)\|_{X \times Y} \leq r\right\}$. For $(u, v) \in B_{r}$, we have

$$
\begin{aligned}
& \left|T_{1}(u, v)(t)\right| \\
\leq & \max _{0 \leq t \leq 1} \mid \int_{0}^{1} G_{1}(t, s)\left[f\left(s, u(s), v(s),{ }^{c} D_{0+}^{\alpha_{1}-1} u(s),{ }^{c} D_{0+}^{\alpha_{2}-1} v(s)\right)-f(s, 0,0,0,0)+f(s, 0,0,0,0)\right] d s \\
& +A_{1}\left[f\left(s, u(s), v(s),{ }^{c} D_{0+}^{\alpha_{1}-1} u(s),{ }^{c} D_{0+}^{\alpha_{2}-1} v(s)\right)-f(s, 0,0,0,0)+f(s, 0,0,0,0)\right] \psi_{1} M \\
& +B_{1}\left[f_{1}\left(s, u(s), v(s),{ }^{c} D_{0+}^{\alpha_{1}-1} u(s),{ }^{c} D_{0+}^{\alpha_{2}-1} v(s)\right)-f(s, 0,0,0,0)+f(s, 0,0,0,0)\right] \varphi_{1}(1) \mid \\
\leq & \left(\max \left\{n_{1}, n_{3}\right\}\|u\|_{X}+\max \left\{n_{2}, n_{4}\right\}\|v\|_{Y}+H_{1}\right)\left(\frac{\varphi_{1}(1)+1}{\Gamma\left(\alpha_{1}+1\right)}+\bar{A}_{1}(1) \psi_{1} M+\bar{B}_{1}(1) \varphi_{1}(1)\right) \\
& \left|T_{1}^{\prime}(u, v)(t)\right| \\
\leq & \max _{0 \leq t \leq 1} \mid \int_{0}^{1} \frac{\partial}{\partial t} G_{1}(t, s)\left[f\left(s, u(s), v(s),{ }^{c} D_{0+}^{\alpha_{1}-1} u(s),{ }^{c} D_{0+}^{\alpha_{2}-1} v(s)\right)-f(s, 0,0,0,0)+f(s, 0,0,0,0)\right] d s \\
& +A_{1}\left[f\left(s, u(s), v(s),{ }^{c} D_{0+}^{\alpha_{1}-1} u(s),{ }^{c} D_{0+}^{\alpha_{2}-1} v(s)\right)-f(s, 0,0,0,0)+f(s, 0,0,0,0)\right] \psi_{1}^{\prime}(t) \\
& +B_{1}\left[f\left(s, u(s), v(s),{ }^{c} D_{0+}^{\alpha_{1}-1} u(s),{ }^{c} D_{0+}^{\alpha_{2}-1} v(s)\right)-f(s, 0,0,0,0)+f(s, 0,0,0,0)\right] \varphi_{1}^{\prime}(t) \mid \\
\leq & \left(\max \left\{n_{1}, n_{3}\right\}\|u\|_{X}+\max \left\{n_{2}, n_{4}\right\}\|v\|_{Y}+H_{1}\right)\left(\bar{e}_{1}+\bar{A}_{1}(1) \frac{1-D_{12}}{\Delta_{1}}+\bar{B}_{1}(1) \frac{1-D_{11}}{\Delta_{1}}\right) .
\end{aligned}
$$


Moreover, we have

$$
\begin{aligned}
& \left|{ }^{c} D_{0+}^{\alpha_{1}-1} T_{1}(u, v)(t)\right| \\
\leq & \max _{0 \leq t \leq 1}\left|\int_{0}^{t} \frac{(t-s)^{1-\alpha_{1}}}{\Gamma\left(2-\alpha_{1}\right)} T_{1}^{\prime}(u, v)(s) d s\right| \\
\leq & \left(\max \left\{n_{1}, n_{3}\right\}\|u\|_{X}+\max \left\{n_{2}, n_{4}\right\}\|v\|_{Y}+H_{1}\right) \frac{1}{\Gamma\left(3-\alpha_{1}\right)}\left(\bar{e}_{1}+\bar{A}_{1}(1) \frac{1-D_{12}}{\Delta_{1}}+\bar{B}_{1}(1) \frac{1-D_{11}}{\Delta_{1}}\right) .
\end{aligned}
$$

Hence we have

$$
\begin{aligned}
& \left\|T_{1}(u, v)(t)\right\|_{X} \\
\leq & \left(\max \left\{n_{1}, n_{3}\right\}\|u\|_{X}+\max \left\{n_{2}, n_{4}\right\}\|v\|_{Y}+H_{1}\right)\left[\left(\frac{\varphi_{1}(1)+1}{\Gamma\left(\alpha_{1}+1\right)}+\bar{A}_{1}(1) \psi_{1^{M}}+\bar{B}_{1}(1) \varphi_{1}(1)\right)\right. \\
& \left.+\frac{1}{\Gamma\left(3-\alpha_{1}\right)}\left(\bar{e}_{1}+\bar{A}_{1}(1) \frac{1-D_{12}}{\Delta_{1}}+\bar{B}_{1}(1) \frac{1-D_{11}}{\Delta_{1}}\right)\right] \\
\leq & \bar{N}_{1} \max \left\{n_{1}, n_{3}\right\}\|u\|_{X}+\bar{N}_{1} \max \left\{n_{2}, n_{4}\right\}\|v\|_{Y}+\bar{N}_{1} H_{1} .
\end{aligned}
$$

Similarly, we can get

$$
\begin{aligned}
\left\|T_{2}(u, v)(t)\right\|_{Y} & =\max _{t \in[0,1]}\left|T_{2}(u, v)(t)\right|+\max _{t \in[0,1]}\left|{ }^{c} D_{0+}^{\alpha_{2}-1} T_{2}(u, v)(t)\right| \\
& \leq \bar{N}_{2} \max \left\{m_{1}, m_{3}\right\}\|u\|_{X}+\bar{N}_{2} \max \left\{m_{2}, m_{4}\right\}\|v\|_{Y}+\bar{N}_{2} H_{2} .
\end{aligned}
$$

Thus, we have

$$
\begin{aligned}
\|T(u, v)(t)\|_{X \times Y} \leq & \left(\bar{N}_{1} \max \left\{n_{1}, n_{3}\right\}+\bar{N}_{2} \max \left\{m_{1}, m_{3}\right\}\right)\|u\|_{X} \\
& +\left(\bar{N}_{1} \max \left\{n_{2}, n_{4}\right\}+\bar{N}_{2} \max \left\{m_{2}, m_{4}\right\}\right)\|v\|_{Y}+\bar{N}_{1} H_{1}+\bar{N}_{2} H_{2} \\
\leq & M_{3}\|(u, v)\|_{X \times Y}+\bar{N}_{1} H_{1}+\bar{N}_{2} H_{2} .
\end{aligned}
$$

Consequently, $\|T(u, v)(t)\|_{X \times Y} \leq r$.

Now for $u_{1}, u_{2} \in X$ and $v_{1}, v_{2} \in Y$, for any $t \in[0,1]$, we get

$$
\begin{aligned}
& \quad\left|T_{1}\left(u_{2}, v_{2}\right)(t)-T_{1}\left(u_{1}, v_{1}\right)(t)\right| \\
& \leq \int_{0}^{1}\left|G_{1}(t, s)\right| \mid f\left(s, u_{2}(s), v_{2}(s),{ }^{c} D_{0+}^{\alpha_{1}-1} u_{2}(s),{ }^{c} D_{0+}^{\alpha_{2}-1} v_{2}(s)\right) \\
& \quad-f\left(s, u_{1}(s), v_{1}(s),{ }^{c} D_{0+}^{\alpha_{1}-1} u_{1}(s),{ }^{c} D_{0+}^{\alpha_{2}-1} v_{1}(s)\right) \mid d s \\
& \quad+\left|A_{1}\left(f_{2}\right)-A_{1}\left(f_{1}\right)\right| \psi_{M}+\left|B_{1}\left(f_{2}\right)-B_{1}\left(f_{1}\right)\right| \varphi_{1}(1) \\
& \leq \max _{s \in[0,1]}\left(n_{1}\left|u_{2}-u_{1}\right|+n_{2}\left|v_{2}-v_{1}\right|+n_{3}\left|{ }^{c} D_{0+}^{\alpha-1} u_{2}-{ }^{c} D_{0+}^{\alpha-1} u_{1}\right|+n_{4}\left|{ }^{c} D_{0+}^{\beta-1} v_{2}-{ }^{c} D_{0+}^{\beta-1} v_{1}\right|\right) \\
& \quad \times\left[\frac{\varphi_{1}(1)+1}{\Gamma(\alpha+1)}+\bar{A}_{1}(1) \psi_{M}+\bar{B}_{1}(1) \varphi_{1}(1)\right]
\end{aligned}
$$




$$
\begin{aligned}
& \left|T_{1}^{\prime}\left(u_{2}, v_{2}\right)(t)-T_{1}^{\prime}\left(u_{1}, v_{1}\right)(t)\right| \\
\leq & \int_{0}^{1} \frac{\partial}{\partial t}\left|G_{1}(t, s)\right| \mid f\left(s, u_{2}(s), v_{2}(s),{ }^{c} D_{0+}^{\alpha_{1}-1} u_{2}(s),{ }^{c} D_{0+}^{\alpha_{2}-1} v_{2}(s)\right) \\
& \quad-f\left(s, u_{1}(s), v_{1}(s),{ }^{c} D_{0+}^{\alpha_{1}-1} u_{1}(s),{ }^{c} D_{0+}^{\alpha_{2}-1} v_{1}(s)\right) \mid d s \\
& \quad+\left|A_{1}\left(f_{2}\right)-A_{1}\left(f_{1}\right)\right| \psi_{1}^{\prime}(t)+\left|B_{1}\left(f_{2}\right)-B_{1}\left(f_{1}\right)\right| \varphi_{1}^{\prime}(t) \\
\leq & \max _{s \in[0,1]}\left(n_{1}\left|u_{2}-u_{1}\right|+n_{2}\left|v_{2}-v_{1}\right|+n_{3}\left|{ }^{c} D_{0+}^{\alpha_{1}-1} u_{2}-{ }^{c} D_{0+}^{\alpha_{1}-1} u_{1}\right|+n_{4}\left|{ }^{c} D_{0+}^{\alpha_{2}-1} v_{2}-{ }^{c} D_{0+}^{\alpha_{2}-1} v_{1}\right|\right) \\
& \times\left[\bar{e}_{1}+\bar{A}_{1}(1) \frac{1-D_{12}}{\Delta_{1}}+\bar{B}_{1}(1) \frac{1-D_{11}}{\Delta_{1}}\right],
\end{aligned}
$$

further,

$$
\begin{aligned}
& \quad\left|{ }^{c} D_{0+}^{\alpha_{1}-1} T_{1}\left(u_{2}, v_{2}\right)(t)-{ }^{c} D_{0+}^{\alpha_{1}-1} T_{1}\left(u_{1}, v_{1}\right)(t)\right| \\
& \leq \max _{s \in[0,1]}\left(n_{1}\left|u_{2}-u_{1}\right|+n_{2}\left|v_{2}-v_{1}\right|+n_{3}\left|{ }^{c} D_{0+}^{\alpha_{1}-1} u_{2}-{ }^{c} D_{0+}^{\alpha_{1}-1} u_{1}\right|+n_{4}\left|{ }^{c} D_{0+}^{\alpha_{2}-1} v_{2}-{ }^{c} D_{0+}^{\alpha_{2}-1} v_{1}\right|\right) \\
& \quad \times \frac{1}{\Gamma\left(3-\alpha_{1}\right)}\left[\bar{e}_{1}+\bar{A}_{1}(1) \frac{1-D_{12}}{\Delta_{1}}+\bar{B}_{1}(1) \frac{1-D_{11}}{\Delta_{1}}\right]
\end{aligned}
$$

and consequently we obtain

$$
\begin{aligned}
& \left\|T_{1}\left(u_{2}, v_{2}\right)-T_{1}\left(u_{1}, v_{1}\right)\right\|_{X} \\
\leq & \max _{s \in[0,1]}\left(n_{1}\left|u_{2}-u_{1}\right|+n_{2}\left|v_{2}-v_{1}\right|+n_{3}\left|{ }^{c} D_{0+}^{\alpha_{1}-1} u_{2}-{ }^{c} D_{0+}^{\alpha_{1}-1} u_{1}\right|+n_{4}\left|{ }^{c} D_{0+}^{\alpha_{2}-1} v_{2}-{ }^{c} D_{0+}^{\alpha_{2}-1} v_{1}\right|\right) \bar{N}_{1} \\
\leq & {\left[\max \left\{n_{1}, n_{3}\right\}\left\|u_{2}-u_{1}\right\|_{X}+\max \left\{n_{2}, n_{4}\right\}\left\|v_{2}-v_{1}\right\|_{Y}\right] \bar{N}_{1} . }
\end{aligned}
$$

Similarly to the above discussion, we can obtain

$$
\begin{aligned}
& \left\|T_{2}\left(u_{2}, v_{2}\right)-T_{2}\left(u_{1}, v_{1}\right)\right\|_{Y} \\
\leq & \max _{s \in[0,1]}\left(m_{1}\left|u_{2}-u_{1}\right|+m_{2}\left|v_{2}-v_{1}\right|+m_{3}\left|{ }^{c} D_{0+}^{\alpha_{1}-1} u_{2}-{ }^{c} D_{0+}^{\alpha_{1}-1} u_{1}\right|+m_{4}\left|{ }^{c} D_{0+}^{\alpha_{2}-1} v_{2}-{ }^{c} D_{0+}^{\alpha_{2}-1} v_{1}\right|\right) \bar{N}_{2} \\
\leq & {\left[\max \left\{m_{1}, m_{3}\right\}\left\|u_{2}-u_{1}\right\|_{X}+\max \left\{m_{2}, m_{4}\right\}\left\|v_{2}-v_{1}\right\|_{Y}\right] \bar{N}_{2}, }
\end{aligned}
$$

Combing (32) with (33), we can get

$$
\begin{aligned}
& \left\|T\left(u_{2}, v_{2}\right)-T\left(u_{1}, v_{1}\right)\right\|_{X \times Y} \\
\leq & \left(\max \left\{n_{1}, n_{3}\right\} \bar{N}_{1}+\max \left\{m_{1}, m_{3}\right\} \bar{N}_{2}\right)\left\|u_{2}-u_{1}\right\|_{X} \\
& +\left(\max \left\{n_{2}, n_{4}\right\} \bar{N}_{1}+\max \left\{m_{2}, m_{4}\right\} \bar{N}_{2}\right)\left\|v_{2}-v_{1}\right\|_{Y} \\
\leq & M_{3}\left(\left\|u_{2}-u_{1}\right\|_{X}+\left\|v_{2}-v_{1}\right\|_{Y}\right) \\
= & M_{3}\left\|\left(u_{2}-u_{1}, v_{2}-v_{1}\right)\right\|_{X \times Y} .
\end{aligned}
$$

Since $M_{3}<1$, the operator $T$ is contractive. By applying the Banach's contraction principle, $T$ has a unique fixed point, which is the unique solution of problem (3) and (4).

Example 1. Consider the following fractional order coupled differential system

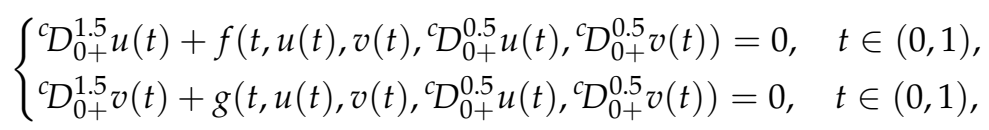


with multi-point and multi-strip boundary conditions

$$
\left\{\begin{array}{l}
u(0)-\sum_{i=1}^{2} \mu_{1 i}{ }^{c} D_{0+}^{0.2} u\left(\theta_{1 i}\right)=\sum_{i=1}^{2} \lambda_{1 i} I_{0+}^{1.5} u\left(\xi_{1 i}\right) \\
u(1)+\sum_{i=1}^{2} \gamma_{1 i}{ }^{c} D_{0+}^{0.2} u\left(\vartheta_{1 i}\right)=\sum_{i=1}^{2} \delta_{1 i} I_{0+}^{1.5} u\left(\eta_{1 i}\right) \\
v(0)-\sum_{i=1}^{2} \mu_{2 i}{ }^{c} D_{0+}^{0.2} v\left(\theta_{2 i}\right)=\sum_{i=1}^{2} \lambda_{2 i} I_{0+}^{1.5} v\left(\xi_{2 i}\right) \\
v(1)+\sum_{i=1}^{2} \gamma_{2 i}{ }^{c} D_{0+}^{0.2} v\left(\vartheta_{2 i}\right)=\sum_{i=1}^{2} \delta_{2 i} I_{0+}^{1.5} v\left(\eta_{2 i}\right)
\end{array}\right.
$$

where

$$
\begin{aligned}
& f\left(t, u(t), v(t),{ }^{c} D_{0+}^{0.5} u(t),{ }^{c} D_{0+}^{0.5} v(t)\right) \\
& =\frac{1}{12(t+1)^{2}} u(t)+\frac{1}{12(t+1)^{2}} v(t)+\frac{1}{12(t+1)^{2}}{ }^{c} D_{0+}^{0.5} u(t)+\frac{1}{12(t+1)^{2}}{ }^{c} D_{0+}^{0.5} v(t)+\frac{1}{12} \\
& \quad g\left(t, u(t), v(t),{ }^{c} D_{0+}^{0.5} u(t),{ }^{c} D_{0+}^{0.5} v(t)\right) \\
& =\frac{1}{8(t+2)^{2}} u(t)+\frac{1}{8(t+2)^{2}} v(t)+\frac{1}{8(t+2)^{2}}{ }^{c} D_{0+}^{0.5} u(t)+\frac{1}{8(t+2)^{2}}{ }^{c} D_{0+}^{0.5} v(t)+\frac{1}{16} \\
& \quad \theta_{j 1}=\vartheta_{j 1}=\lambda_{j 1}=\frac{1}{2}, \xi_{j 1}=\eta_{j 1}=\frac{1}{3}, \mu_{j 1}=\frac{1}{8}, \gamma_{j 1}=\frac{1}{4}, \delta_{j 1}=\frac{1}{3}, \\
& \theta_{j 2}=\vartheta_{j 2}=\lambda_{j 2}=\frac{3}{4}, \xi_{j 2}=\eta_{j 2}=\frac{5}{6}, \mu_{j 2}=\frac{1}{16}, \gamma_{j 2}=\frac{1}{3}, \delta_{j 2}=\frac{1}{5}, j=1,2 .
\end{aligned}
$$

It is easy to see that $\alpha_{1}=\alpha_{2}=p_{1}=p_{2}=1.5, q_{1}=q_{2}=0.2$ and

$$
\begin{aligned}
& \left|f\left(t, u, v,{ }^{c} D_{0+}^{\alpha_{1}-1} u,{ }^{c} D_{0+}^{\alpha_{2}-1} v\right)\right| \leq \frac{1}{12}+\frac{1}{12}|u|+\frac{1}{12}|v|+\frac{1}{12}\left|{ }^{c} D_{0+}^{\alpha_{1}-1} u\right|+\frac{1}{12}\left|{ }^{c} D_{0+}^{\alpha_{2}-1} v\right|, \\
& \left|g\left(t, u, v,{ }^{c} D_{0+}^{\alpha_{1}-1} u,{ }^{c} D_{0+}^{\alpha_{2}-1} v\right)\right| \leq \frac{1}{16}+\frac{1}{16}|u|+\frac{1}{16}|v|+\frac{1}{16}\left|{ }^{c} D_{0+}^{\alpha_{1}-1} u\right|+\frac{1}{16}\left|{ }^{c} D_{0+}^{\alpha_{2}-1} v\right| .
\end{aligned}
$$

Hence, condition $\left(H_{5}\right)$ holds. Further, we have $D_{j 1}=0.5016, D_{j 2}=0.1627, E_{j 1}=0.2831, E_{j 2}=0.3939$ $j=1,2$ and

$$
\begin{aligned}
& \left|f\left(t, u_{1}, u_{2}, u_{3}, u_{4}\right)-f\left(t, v_{1}, v_{2}, v_{3}, v_{4}\right)\right| \\
\leq & \frac{1}{12}\left|u_{1}-v_{1}\right|+\frac{1}{12}\left|u_{2}-v_{2}\right|+\frac{1}{12}\left|u_{3}-v_{3}\right|+\frac{1}{12}\left|u_{4}-v_{4}\right|, \\
& \left|g\left(t, u_{1}, u_{2}, u_{3}, u_{4}\right)-g\left(t, v_{1}, v_{2}, v_{3}, v_{4}\right)\right| \\
\leq & \frac{1}{16}\left|u_{1}-v_{1}\right|+\frac{1}{16}\left|u_{2}-v_{2}\right|+\frac{1}{16}\left|u_{3}-v_{3}\right|+\frac{1}{16}\left|u_{4}-v_{4}\right| .
\end{aligned}
$$

So condition $\left(\mathrm{H}_{6}\right)$ holds.

By simple computation, for $j=1,2$, we have $\Delta_{j}=0.9318, \varphi_{j}(1)=0.8387, \psi_{j^{M}}=1.4959, \bar{e}_{j}=1.5307$ and

$$
\begin{aligned}
N_{j} & =\frac{\varphi_{j}(1)+1}{\Gamma\left(\alpha_{j}+1\right)}+\bar{A}_{j}(1) \psi_{j}+\bar{B}_{j}(1) \varphi_{j}(1) \\
& +\frac{1}{\Gamma\left(3-\alpha_{j}\right)}\left(\bar{e}_{j}+\bar{A}_{j}(1) \frac{1-D_{j 2}}{\Delta_{j}}+\bar{B}_{j}(1) \frac{1-D_{j 1}}{\Delta_{j}}\right)=4.8858 .
\end{aligned}
$$


Therefore,

$$
\begin{aligned}
& N_{1} \max \left\{\rho_{1}, \rho_{3}\right\}+N_{2} \max \left\{\varrho_{1}, \varrho_{3}\right\} \approx 0.7125<1, \\
& N_{1} \max \left\{\rho_{2}, \rho_{4}\right\}+N_{2} \max \left\{\varrho_{2}, \varrho_{4}\right\} \approx 0.7125<1 .
\end{aligned}
$$

By Theorem 3, boundary value problem (35) and (36) has at least one solution. We also have

$$
\begin{aligned}
& N_{1} \max \left\{n_{1}, n_{3}\right\}+N_{2} \max \left\{m_{1}, m_{3}\right\} \approx 0.7125<1, \\
& N_{1} \max \left\{n_{2}, n_{4}\right\}+N_{2} \max \left\{m_{2}, m_{4}\right\} \approx 0.7125<1 .
\end{aligned}
$$

By Theorem 4, boundary value problem (35) and (36) has a unique solution.

In Example 1, by applying the Leray-Schauder's Alternative and Banach's constraction principle, we establish the existence and uniqueness conclusions to problem (35) and (36), respectively. It is important to note that this example is not fixed, it represents a class of models. Both the form of the nonlinear terms in (35) and the parameters of boundary condition (36) can be adjusted, only satisfied H5 and H6.

\section{Conclusions}

First of all, subject to the coupled system of second-order differential equations with nonlinearities depending on two unknown functions as well as their derivatives, by defining the appropriate upper and lower solutions, combining with Nagumo conditions, the truncation function is constructed successfully. It is proved that there exists a solution to this truncation system that is just between the upper and lower solutions, and the derivative of the solution is bounded. It means the solution of the truncation system is the one of the original problem.

Secondly, we extend the coupled system of second-order differential equations to a coupled system of Caputo fractional differential equations. To the differential model with wide application background, the existence and uniqueness results of solutions are investigated by using the Leray-Schauder's alternative and the Banach's contraction principle.

However, to the best of our knowledge, this technique of upper and lower solutions has not been applied yet to the differential system with mixed fractional order derivative definition, which will be the direction of our further research.

Author Contributions: All authors contributed equally to the manuscript, read and approved the final draft. All authors have read and agreed to the published version of the manuscript.

Funding: This research received no external funding.

Acknowledgments: The authors thank the anonymous reviewers for their careful reading and constructive comments.

Conflicts of Interest: The authors declare no conflict of interest.

\section{References}

1. Podlubny, I. Fractional Differential Equations; Academic Press: San Diego, CA, USA, 1999.

2. Oldham, K.; Spainer, J. The Fractional Calculus; Academic Press: New York, NY, USA, 1974.

3. Miller, K.; Ross, B. An Introduction to the Fractional Calculus and Fractional Differential Equations; Wiley-InterScience: New York, NY, USA, 1993.

4. Hilfer, R. Application of Fractional Calculus in Physics; World Scientific: Singapore, 2000.

5. Asif, N.A.; Khan, R.A. Positive solutions to singular system with four-point coupled boundary conditions. J. Math. Anal. Appl. 2012, 386, 848-861. [CrossRef]

6. Zhu, C.; Zhang, X.; Wu, Z. Solvability for a coupled system of fractional differential equations with integral boundary conditions. Taiwanese J. Math. 2013, 17, 2039-2054. [CrossRef]

7. Agarwal, R.P.; Ahmad, B.; Garout, D.; Alsaedi, A. Existence results for coupled nonlinear fractional differential equations equipped with nonlocal coupled flux and multi-point boundary conditions. Chaos Solitons Fractals 2017, 102, 149-161. [CrossRef] 
8. Ahmad, B.; Alsaedi, A.; Aljoudi, S.; Ntouyas, S.K. On a coupled system of sequential fractional differential equations with variable coefficients and coupled integral boundary conditions. Bull. Math. Soc. Sci. Math. Roum. 2017, 60, 3-18.

9. Sun, Z.; Ge, W.; Li, L. Multiple periodic orbits of high-dimensional differential delay systems. Adv. Differ. Equ. 2019, 2019, 488. [CrossRef]

10. Jiao, L.; Zhang, X. Multi-parameter second-order impulsive indefinite boundary value problems. Adv. Differ. Equ. 2018, 2018, 158. [CrossRef]

11. Zhang, X.; Feng, M. Nontrivial convex solutions on a parameter of impulsive differential equation with Monge-Ampère operator. Bound. Value Probl. 2017, 2017, 172. [CrossRef]

12. Ge, W.; Zhang, L. Multiple periodic solutions of delay differential systems with $2 k-1$ lags via variational approach. Discret. Contin. Dyn. Syst. 2016, 36, 4925-4943. [CrossRef]

13. Cui, Y.; Zou, Y. Monotone iterative method for differential systems with coupled integral boundary value problems. Bound. Value Probl. 2012, 2013, 245. [CrossRef]

14. Liu, X.; Jia, M. The method of lower and upper solutions for the general boundary value problems of fractional differential equations with p-Laplacian. Adv. Differ. Equ. 2018, 2018, 28. [CrossRef]

15. Gao, Y.; Chen, P. Existence of solutions for a class of nonlinear higher-order fractional differential equation with fractional nonlocal boundary condition. Adv. Differ. Equ. 2016, 2016, 314. [CrossRef]

16. Guo, L.; Liu, L.; Wu, Y. Uniqueness of iterative positive solutions for the singular fractional differential equations with integral boundary conditions. Bound. Value Probl. 2016, 2016, 147. [CrossRef]

17. Xie, W.; Xiao, J.; Luo, Z. Existence of Solutions for Riemann-Liouville Fractional Boundary Value Problem. Abstract Appl. Anal. 2014, 2014, 540351. [CrossRef]

18. Cabada, A. The method of lower and upper solutions for second, third, fourth and higher order boundary value problem. J. Math. Anal. Appl. 1994, 185, 302-320. [CrossRef]

19. Ehme, J.; Eloe, P. W.; Henderson, J. Upper and lower solution methods for fully nonlinear boundary value problems. J. Differ. Equ. 2001, 180, 51-64. [CrossRef]

20. Lin, L.; Liu, X.; Fang, H. Method of upper and lower solutions for fractional differential equations. Electron. J. Differ. Equ. 2012, 100, 596-602.

21. Bai, Z.; Zhang, S.; Sun, S.; Yin, C. Monotone iterative method for fractional differential equations. Electron. J. Differ. Equ. 2016, 6, 1-8.

22. Liu, X.; Jia, M.; Ge, W. The method of lower and upper solutions for mixed fractional four-point boundary value problem with p-Laplacian operator. Appl. Math. Lett. 2017, 65, 56-62. [CrossRef]

23. Sheng, K.; Zhang, W.; Bai, Z. Positive solutions to fractional boundaryvalue problems with p-Laplacian on time scales. Bound. Value Probl. 2018, 2018, 70. [CrossRef]

24. Tian, Y.; Sun, S.; Bai, Z. Positive solutions of fractional differential equations with p-Laplacian. J. Funct. Space. 2017, 2017, 3187492. [CrossRef]

25. Li, L.; Sun, H.; Ge, W. Multiple periodic solutions of high order differential delay equations with $2 k-1$ lags. Adv. Differ. Equ. 2019, 2019, 3 [CrossRef]

26. Li, L.; Sun, H.; Ge, W. On the Number of Periodic Solutions to Kaplan-Yorke-like High Order Differential Delay Equations with 2k Lags. Int. J. Bifur. Chaos 2019, 29, 1950196. [CrossRef]

27. Sokolov, I.M.; Klafter, J.; Blumen, A. Fractional kinetics. Phys. Today. 2002, 55, 48-54. [CrossRef]

28. Javidi, M.; Ahmad, B. Dynamic analysis of time fractional order phytoplankton-toxic phytoplanktonzooplankton system. Ecol. Model. 2015, 318, 8-18. [CrossRef]

29. Petras, I.; Magin, R.L. Simulation of drug uptake in a two compartmental fractional model for a biological system. Commun. Nonlinear Sci. Number. Simul. 2011, 16, 4588-4595. [CrossRef]

30. Ding, Y.; Wang, Z.; Ye, H. Optimal control of a fractional-order HIV-immune system with memory. IEEE Trans. Control Syst. Technol. 2012, 20, 763-769. [CrossRef]

31. Aam, A.; Rida, S.Z.; Khalil, M. Fractional modeling dynamics of HIV and CD4+T-cells during primary infection. Nonlinear Biomed. Phys. 2012, 6, 1.

32. Ahmad, B.; Ntouyas, S.K. A fully hadamard type integral boundary value problem of a coupled system of fractional differential equations. Fract. Calc. Appl. Anal. 2014, 2, 348-360. [CrossRef]

33. Li, Y.; Sang, Y.; Zhang, H. Solvability of a coupled system of nonlinear fractional differential equations with fractional integral conditions. J. Appl. Math. Comput. 2015, 50,1-19. [CrossRef] 
34. Cui, M.; Zhu ,Y.; Pang, H. Existence and uniqueness results for a coupled fractional order systems with the multi-strip and multi-point mixed boundary conditions. Adv. Differ. Equ. 2017, 2017, 224. [CrossRef]

35. Kilbas, A.A.; Srivastava, H.M.; Trujillo, J.J. Theory and Applications of Fractional Differential Equations; Elsevier: Amsterdam, The Netherlands, 2006.

(c) 2020 by the authors. Licensee MDPI, Basel, Switzerland. This article is an open access article distributed under the terms and conditions of the Creative Commons Attribution

(CC BY) license (http://creativecommons.org/licenses/by/4.0/). 